\title{
Entropy-Like Properties and $\mathfrak{L}_{q}$-Norms of Hypergeometric Orthogonal Polynomials: Degree Asymptotics
}

\author{
Jesús S. Dehesa ${ }^{1,2}$ (D)
}

check for

updates

Citation: Dehesa, J.S. Entropy-Like Properties and $\mathfrak{L}_{q}$-Norms of Hypergeometric Orthogonal Polynomials: Degree Asymptotics. Symmetry 2021, 13, 1416.

https://doi.org/10.3390/sym13081416

Academic Editor: Paolo Emilio Ricci

Received: 26 May 2021

Accepted: 13 July 2021

Published: 3 August 2021

Publisher's Note: MDPI stays neutral with regard to jurisdictional claims in published maps and institutional affiliations.

Copyright: (c) 2021 by the authors. Licensee MDPI, Basel, Switzerland. This article is an open access article distributed under the terms and conditions of the Creative Commons Attribution (CC BY) license (https:/ / creativecommons.org/licenses/by/ $4.0 /)$.
1 Instituto Carlos I de Física Teórica y Computacional, Universidad de Granada, 18071 Granada, Spain; dehesa@ugr.es

2 Departamento de Física Atómica, Molecular y Nuclear, Universidad de Granada, 18071 Granada, Spain

\begin{abstract}
In this work, the spread of hypergeometric orthogonal polynomials (HOPs) along their orthogonality interval is examined by means of the main entropy-like measures of their associated Rakhmanov's probability density—so, far beyond the standard deviation and its generalizations, the ordinary moments. The Fisher information, the Rényi and Shannon entropies, and their corresponding spreading lengths are analytically expressed in terms of the degree and the parameter(s) of the orthogonality weight function. These entropic quantities are closely related to the gradient functional (Fisher) and the $\mathfrak{L}_{q}$-norms (Rényi, Shannon) of the polynomials. In addition, the degree asymptotics for these entropy-like functionals of the three canonical families of HPOs (i.e., Hermite, Laguerre, and Jacobi polynomials) are given and briefly discussed. Finally, a number of open related issues are identified whose solutions are both physico-mathematically and computationally relevant.
\end{abstract}

Keywords: special functions; classical orthogonal polynomials; entropy-like measures; asymptotics

\section{Introduction}

Hypergeometric orthogonal polynomials (HOPs), also called Shohat-Favard polynomials and classical orthogonal polynomials, play a key role in the development of the theory of special functions, and they are instrumental in numerous scientific problems, ranging from approximation theory to quantum theory and mathematical physics [1-8]. Here, we examine and review the knowledge of the spreading measures of the HOPs in a real continuous variable, $\left\{p_{n}(x)\right\}$, orthogonal with respect to the weight function $h(x)$ on the interval $\Lambda \subseteq \mathbb{R}$. These quantities measure the different spreading facets of their associated probability density as follows:

$$
\rho_{n}(x)=\hat{p}_{n}^{2}(x) h(x)=\frac{1}{\kappa_{n}} p_{n}^{2}(x) h(x), \quad \text { with } \quad \kappa_{n}=\int_{\Lambda}\left|p_{n}(x)\right|^{2} h(x) d x,
$$

where the symbol $\hat{p}_{n}(x)=p_{n}(x) / \kappa_{n}^{\frac{1}{2}}$ denotes the orthonormal polynomial. This quantity is called Rakhmanov's density [9] because he first discovered it to govern the asymptotical $(n \rightarrow+\infty)$ behavior of the ratio of two polynomials with consecutive orders. Later on, it was realized that this normalized-to-unity probability density characterizes the Born's probability density of the bound stationary states of a great deal of quantum systems in one and many dimensions (harmonic oscillator, hydrogenic atoms, etc.) [3,10,11]. This is because the physical solutions (wave functions) of the Schrödinger equation for a broad family of quantum potentials, such as the spherically symmetric ones $[3,12,13]$, are controlled by the three canonical HOPs families of Hermite $H_{n}(x)$, Laguerre $L_{n}^{(\alpha)}(x)$ and Jacobi $P_{n}^{(\alpha, \beta)}(x)$ types, respectively. So, the spreading measures of the HOPs quantify numerous physical and chemical properties of quantum systems, such as the physical entropy-like measures, which are the basic variables of the classical and quantum information theories [14-16]. These spreading measures include standard deviation (and its generalizations, 
the ordinary moments) and the entropy-like measures (Fisher information [17,18], Shannon entropy $[14,19]$ and Rényi entropies $[20,21])$ and $\mathfrak{L}_{q}$-norms of the density $\rho_{n}(x)$. In order for all these quantifiers to be mutually compared on the same footing, we have examined the corresponding information-theoretic lengths of Fisher, Shannon and Rényi types [22-25]. These quantities, together with the standard deviation, are direct spreading measures [26] of Rakhmanov's density because they have some common properties: namely, the same units as the variable $x$, invariance under translations and reflections, linear scaling with $x$ and vanishing in the limit as $\rho_{n}(x)$ approaches a delta function. Note that, contrary to the standard deviation, which measures the concentration of the probability density around a particular point (the centroid) of the orthogonality interval, the information-theoretic lengths do not refer to any specific point of that interval.

The Fisher information $F(\rho)$ is a functional of the derivative of the density $\rho(x)$; so, it is sensitive to the fluctuations of the density. The Fisher information controls the localization of the density around its nodes, appropriately grasping the oscillatory nature of the density; this confers it a relevant role in the characterization of a great diversity of scientific phenomena [18]. It is a local spreading measure of the density, which quantifies the pointwise concentration of $\rho$. Moreover, the higher this quantity, the more localized the density, and the higher the accuracy in predicting the localization of the particle.

The Rényi entropies $R_{q}[\rho], 0<q<\infty, q \neq 1$, conform a family of spreading measures of the density $\rho(x)$, depending on a real parameter $q$. They are $q$-power functionals of the density, closely related to the $\mathfrak{L}_{q}$-norms, so they have a global character, contrary to the previous Fisher information. According to the values of the parameter $q$, these quantities supply complementary ways to quantify the different facets of the extent/shape of $\rho(x)$ all over the orthogonality interval, including the Onicescu functional [27] or disequilibrium $\mathcal{D}[\rho]=\exp \left(-R_{2}[\rho]\right)$ and the Shannon entropy $S[\rho]=\lim _{q \rightarrow 1} R_{q}[\rho]$. These quantities satisfy a large number of interesting physico-mathematical properties [14,28-31]. In particular, the Shannon entropy is the only one that satisfies all the hypotheses of Shannon's theorem of information theory [14,19] as well as some other important criteria [32]; for instance, Shannon entropy becomes thermodynamical entropy in the case of a thermal ensemble. It is worth noting that $S[\rho]$ can have any values in $[-\infty, \infty]$, contrary to differential Shannon entropy, $-\sum_{i} p_{i} \ln p_{i}$, of a probability on a discrete sample space, which is always positive. Moreover, any sharp peaks in $\rho(x)$ tends to make $S[\rho]$ negative, whereas a slowly decaying density's tail provokes positive values for $S[\rho]$; hence, the Shannon entropy $S[\rho]$ estimates the total extent of the density $\rho$.

In this work, we update the analytical determination of the spreading measures of the HOPs with emphasis on the entropy-like quantities and $\mathfrak{L}_{q}$-norms because of their relevance in the information theory of special functions and quantum systems, and to facilitate its numerical and symbolic computation. We should keep in mind that the naive numerical evaluation of these quantities using quadratures is often not convenient due to the increasing number of integrable singularities when the polynomial degree $n$ is increasing, which spoils any attempt to achieve reasonable accuracy, even for rather small $n$. In addition, the asymptotics $(n \rightarrow \infty)$ of these polynomial functionals has also been considered, although briefly discussed. The degree asympotics of HOPs and its generalizations was initiated in the middle of the nineties by A.I. Aptekarev, J.S. Dehesa, W. van Assche and their collaborators [33-35] and reviewed with some physical and mathematical applications in 2001 [11] and 2010 [36], respectively. The $q$-asymptotics and the weight-function's parameter asymptotics for unweighted and weighted $\mathfrak{L}_{q}$-norms are not considered here. The degree asymptotics was recently used to evaluate physical Rényi and Shannon entropies for the highly-excited (Rydberg) states of quantum systems of harmonic (oscillator-like) [37,38] and Coulombic (hydrogenic-like) types [39-41] as well as for Rydberg atoms, which are used as building elements of logical gates in quantum computation.

Here, we do not consider the entropy-like measures of polynomials with varying weights (i.e., polynomials whose weight-function's parameter does depend on the polynomial degree), which are also of great mathematical and physical interest [42,43], nor do we 
consider another kind of entropy, the discrete Shannon entropy of HOPs [44] introduced in 2009, which was explicitly calculated for Chebyshev polynomials and whose degree asymptotics for Jacobi polynomials was determined in 2015 [45] (see also [46] for the discrete Tsallis and Rényi entropies).

This paper has the following structure. In Section 2, we give the spreading measures for an arbitrary probability density $\rho(x)$ of dispersion (standard deviation and ordinary moments) and information-theoretic (Fisher, Shannon and Rényi) types, which are considered here. In Section 3, the moments around the origin and the standard deviation for the three canonical families of real HOPs (namely, Hermite, Laguerre and Jacobi) are explicitly calculated. In Sections 4 and 5, we determine the Fisher and Rényi (and the associated weighted $\mathfrak{L}_{q}$-norms) spreading measures of the HOPs for all degree $n$, respectively. In Sections 6-8, we calculate the degree asymptotics of the weighted and generalized weighted $\mathfrak{L}_{q}$-norms for Hermite, Laguerre and general (i.e., Szegö type) orthonormal polynomials and Jacobi polynomials, respectively. In Sections 9 and 10 we show the Shannon spreading measures of the HOPs and their degree asymptotics via the appropriate (unweighted, weighted) $\mathfrak{L}_{q}$-norms, respectively. Finally, some concluding remarks are pointed out and a number of open related issues are identified.

\section{Spreading Measures of a Probability Density}

In this section, we describe the spreading measures of dispersion (ordinary moments, standard deviation) and information-theoretic (Fisher information, Shannon entropy, Rényi entropies) types for a random variable $X$ characterized by the continuous probability density $\rho(x), x \in \Lambda \subseteq \mathbb{R}$.

To estimate the spread of $X$ over the interval $\Lambda$, we often take a familiar dispersion measure [47] related to the moments around a particular point of $\Lambda$; usually, one chooses the origin (ordinary moments or moments around the origin $\left\langle x^{k}\right\rangle$ ) or the centroid $\langle x\rangle$ (central moments or moments around the centroid $\left\langle(x-\langle x\rangle)^{k}\right\rangle$ ), or we use some information-theoretic quantities connected with the frequency or entropic moments $W_{q}[\rho]=\left\langle[\rho(x)]^{q-1}\right\rangle=\left\|\rho_{n}\right\|_{q}^{q}[47-49]$, which do not depend on any particular point of the interval and are closely related to the $\mathfrak{L}_{q}$-norms of the density. Each set of moments determines the probability density $\rho(x)$ under certain conditions as the associated ordinary [50] and entropic moment problems state. The expectation value of $f(x)$ with respect to the (normalized-to-unity) density $\rho(x)$ is given by $\langle f(x)\rangle=\int_{\Lambda} f(x) \rho(x) d x$. The most familiar dispersion measure is the statistical root-mean-square or standard deviation $\Delta x$, which is the square root of the variance:

$$
V[\rho]=(\Delta x)^{2}=\left\langle x^{2}\right\rangle-\langle x\rangle^{2}
$$

The entropic moments quantify the extent/shape to which the probability is, in fact, distributed. They are, at times, much better probability quantifiers than the ordinary moments [48]; moreover, they are fairly efficient in the range where the ordinary moments are fairly inefficient [51]. Two relevant spreading measures related to the entropic moments are the entropy-like measures of Rényi and Shannon types. The Rényi entropies $R_{q}[\rho]$ of $\rho(x)$ are defined as follows $[20,21]$ :

$$
R_{q}[\rho]=\frac{1}{1-q} \ln W_{q}[\rho]=\frac{1}{1-q} \ln \int_{\Lambda}[\rho(x)]^{q} d x, \quad q>0, \quad q \neq 1,
$$

The Shannon entropy [14,19] is given by the limiting value $q \rightarrow 1$, taking into account the normalization condition $W_{1}[\rho]=1$ as follows:

$$
S[\rho]=\lim _{q \rightarrow 1} R_{q}[\rho]=-\int_{\Lambda} \rho(x) \ln \rho(x) d x .
$$

The Rényi parameter $q$ allows to enhance or diminish the contribution of the integrand over different regions to the whole integral in (2). Higher values of $q$ make the function 
$[\rho(x)]^{q}$ to concentrate around the local maxima of the distribution, while the lower values have the effect of smoothing that function over its whole domain. It is in this sense that the parameter $q$ provides a powerful tool in order to obtain information on the configuration shape of the probability density by means of the Rényi entropies. Moreover, let us mention here the monotonicity relations given by the following:

$$
R_{p}[\rho] \geq R_{q}[\rho], \quad \text { if } \quad p \leq q ; \quad \text { and } \quad \frac{p-1}{p} R_{p}[\rho] \geq \frac{q-1}{q} R_{q}[\rho], \quad \text { if } \quad p \geq q>1
$$

which, in particular, allow one to lowerbound all the Rényi entropies by means of the second-order entropy as $R_{q}[\rho] \geq \frac{1}{2} R_{2}[\rho]$, for $q>0$.

A third remarkable, qualitatively different, entropy-like spreading measure of $\rho(x)$ is the Fisher information $[17,18]$ which is defined as follows:

$$
F[\rho]:=\int_{\Lambda} \frac{\left[\rho^{\prime}(x)\right]^{2}}{\rho(x)} d x
$$

Opposite to the previous dispersion and entropy-like entropies, which have a global character because they are power and logarithmic functionals of the density, the Fisher information has a locality property because it is a functional of the derivative of $\rho(x)$. Moreover, these three information-theoretic spreading measures (Shannon, Rényi, Fisher) do not depend on any particular point of the interval $\Lambda$, contrary to the standard deviation. However, they have different units from the standard deviation (i.e., the units of $X$ ) so that they can not be mutually compared. To overcome this difficulty, the following informationtheoretic lengths were introduced [26]:

$$
\begin{gathered}
\mathcal{L}_{q}^{R}[\rho]=e^{R_{q}[\rho]}=\left(W_{q}[\rho]\right)^{\frac{1}{1-q}}=\left\{\int_{\Lambda}[\rho(x)]^{q} d x\right\}^{\frac{1}{1-q}}, \quad \text { Rényi length, } \\
\mathcal{L}_{1}^{S}[\rho]=\lim _{q \rightarrow 1} \mathcal{L}_{q}^{R}[\rho]=\exp (S[\rho])=\exp \left\{-\int_{\Lambda} \rho(x) \ln \rho(x) d x\right\}, \quad \text { Shannon length, } \\
\delta x=\frac{1}{\sqrt{F[\rho]}}=\left\{\int_{\Lambda} \frac{\left[\rho^{\prime}(x)\right]^{2}}{\rho(x)} d x\right\}^{-\frac{1}{2}}, \quad \text { Fisher length. }
\end{gathered}
$$

We remark that the quantities $\left(V[\rho], R_{q}[\rho], S[\rho], F[\rho]\right)$, and its related spreading lengths $\left(\Delta x, \mathcal{L}_{q}^{R}[\rho], \mathcal{L}_{1}^{S}[\rho], \delta x\right)$, are complementary since each of them grasps a single different facet of the probability density $\rho(x)$. So, the variance measures the concentration of the density around the centroid, while the Rényi and Shannon entropies are measures of the configurational aspects of the extent/shape to which the density is, in fact, concentrated, and the Fisher information is a quantifier of the oscillatory character of the density since it estimates the pointwise concentration of the probability over its support $\Lambda$. All spreading lengths share the following properties $[26,52,53]$ : same units as the random variable, translation, reflection invariance and linear scaling under adequate boundary conditions, vanishing when the density tends to a delta function. Moreover, they fulfill an uncertainty property [24,54-56] and the Cramér-Rao [52] and Shannon [19] inequalities given by the following:

$$
\delta x \leq \Delta x, \quad \text { and } \quad \mathcal{L}_{1}^{S}[\rho] \leq(2 \pi e)^{\frac{1}{2}} \Delta x,
$$

respectively.

In the next sections, we determine the previous spreading measures for the Rakhmanov density (1) of the real hypergeometric polynomials $\left\{p_{n}(x)\right\}$, orthogonal with respect to the weight function $h(x)$ on the interval $\Lambda$ so that the following holds:

$$
\int_{\Lambda} p_{n}(x) p_{m}(x) h(x) d x=\kappa_{n} \delta_{n, m}, \quad \operatorname{deg} p_{n}=n
$$


where the weight function has the following expressions:

$$
\begin{aligned}
h_{H}(x) & =e^{-x^{2}}, \quad x \in(-\infty,+\infty) \\
h_{\alpha}^{L}(x) & =x^{\alpha} e^{-x}, \quad(\alpha>-1), \quad x \in[0,+\infty) \\
h_{\alpha, \beta}^{J}(x) & =(1-x)^{\alpha}(1+x)^{\beta}, \quad(\alpha, \beta>-1), \quad x \in[-1,+1]
\end{aligned}
$$

for the HOPs families of Hermite $H_{n}(x)$, Laguerre $L_{n}^{(\alpha)}(x)$ and Jacobi $P_{n}^{(\alpha, \beta)}(x)$ types, respectively. The corresponding normalization constants are as follows:

$$
\begin{gathered}
\kappa_{n}^{H} \equiv d_{n}^{2}=\sqrt{\pi} n ! 2^{n}, \\
\kappa_{n}^{L} \equiv d_{n, \alpha}^{2}=\Gamma(n+\alpha+1) / n !, \\
\kappa_{n}^{J} \equiv d_{n, \alpha, \beta}^{2}=\frac{2^{\alpha+\beta+1} \Gamma(\alpha+n+1) \Gamma(\beta+n+1)}{n !(\alpha+\beta+2 n+1) \Gamma(\alpha+\beta+n+1)},
\end{gathered}
$$

respectively. Note that $\kappa_{n}=1$ for the orthonormal polynomials $\hat{p}_{n}(x)$ of Hermite $\hat{H}_{n}(x)$, Laguerre $\hat{L}_{n}^{(\alpha)}(x)$ and Jacobi $\hat{P}_{n}^{(\alpha, \beta)}(x)$ types.

\section{Ordinary Moments and Standard Deviation of HOPs}

In this section, we show the ordinary moments and the standard deviation for the three canonical families of the hypergeometric orthonormal polynomials defined by the expressions (10) and (11). They are given by the corresponding quantities of the associated Rakhmanov density given by (1):

$$
\left\langle x^{k}\right\rangle_{n}=\int_{a}^{b} x^{k} \rho_{n}(x) d x=\frac{1}{\kappa_{n}} \int_{a}^{b} x^{k} p_{n}^{2}(x) h(x) d x \quad k=0,1,2, \ldots
$$

for the ordinary moments, and

$$
(\Delta x)_{n}=\left(\left\langle x^{2}\right\rangle_{n}-\langle x\rangle_{n}^{2}\right)^{\frac{1}{2}},
$$

for the standard deviation. These quantities can be obtained from the different characterizations $[3,4,6]$ of the HOPs, such as the explicit expression [22-24], the orthogonality relation (10), the three-term recurrence relation [57] and the second-order differential equation $[3,58]$. Briefly, for Hermite polynomials these quantities have the following values:

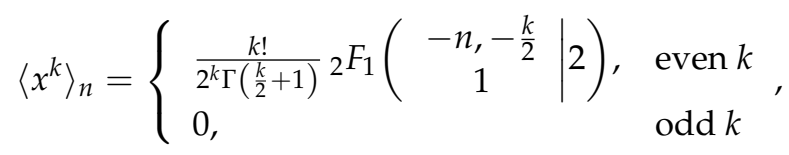

where ${ }_{2} F_{1}(a, b ; c ; 2)$ denotes the Gauss hypergeometric function ${ }_{2} F_{1}(a, b ; c ; x)$ [6] evaluated at $x=2$ for the ordinary moments, and

$$
(\Delta x)_{n}=\sqrt{n+\frac{1}{2}}
$$

for the standard deviation of the orthonormal Hermite polynomials. For Laguerre polynomials the following values

$$
\begin{gathered}
\left\langle x^{k}\right\rangle_{n, \alpha}=\frac{n ! \Gamma(k+\alpha+1)}{\Gamma(n+\alpha+1)} \sum_{r=0}^{n}\left(\begin{array}{c}
k \\
n-r
\end{array}\right)^{2}\left(\begin{array}{c}
k+\alpha+r \\
r
\end{array}\right), \\
(\Delta x)_{n, \alpha}=\sqrt{2 n^{2}+2(\alpha+1) n+\alpha+1},
\end{gathered}
$$


were found for the ordinary moments and for the standard deviation of the orthonormal Laguerre polynomials, respectively. For Jacobi polynomials, one has the following expressions:

$$
\begin{aligned}
\left\langle x^{k}\right\rangle_{n, \alpha, \beta} & =\sum_{i=n-k}^{n}\left(\sum_{t=i}^{n}(-1)^{t-i}\left(\begin{array}{l}
n \\
t
\end{array}\right) \frac{\Gamma(\alpha+n+1) \Gamma(\alpha+\beta+n+t+1)}{n ! 2^{t} \Gamma(\alpha+\beta+n+1) \Gamma(\alpha+t+1)}\left(\begin{array}{l}
t \\
i
\end{array}\right)\right) \\
& \times \frac{(-1)^{k+i-n} 2^{n}(k+i) ! \Gamma(n+\alpha+\beta+1)}{(k+i-n) ! \Gamma(2 n+\alpha+\beta+1)} \\
& \times{ }_{2} F_{1}\left(\begin{array}{c|c}
n-k-i, n+\beta+1 & 2 \\
2 n+\alpha+\beta+2 & 2
\end{array}\right) .
\end{aligned}
$$

for the ordinary moments of the orthonormal Jacobi polynomials, and

$$
\begin{aligned}
& (\Delta x)_{n, \alpha, \beta}=\left[\frac{4(n+1)(n+\alpha+1)(n+\beta+1)(n+\alpha+\beta+1)}{(2 n+\alpha+\beta+1)(2 n+\alpha+\beta+2)^{2}(2 n+\alpha+\beta+3)}\right. \\
& \left.+\frac{4 n(n+\alpha)(n+\beta)(n+\alpha+\beta)}{(2 n+\alpha+\beta-1)(2 n+\alpha+\beta)^{2}(2 n+\alpha+\beta+1)}\right]^{1 / 2},
\end{aligned}
$$

for the standard deviation. Note, in particular, that the asymptotical behavior of the standard deviation of the HOPs goes as $\sqrt{n}$ (Hermite), $\sqrt{2} n$ (Laguerre) and $1 / \sqrt{2}$ (Jacobi) when the weight's parameters are fixed. Finally, it is worth mentioning here that other kinds of moments of orthogonal polynomials were recently considered, such as the exponential and logarithmic moments as well as the generalized Krein-like moments of HOPs [59].

\section{Fisher's Spreading Length of HOPs}

In this section, we give the values of the Fisher spreading length (8) for the three canonical families of the HOPs defined by the expressions (10) and (11). They are given by the corresponding quantities of the associated Rakhmanov probability density $\rho_{n}(x)$ given by (1):

$$
(\delta x)_{n} \equiv \frac{1}{\sqrt{F\left[\rho_{n}\right]}} \equiv\left\langle\left[\frac{d}{d x} \ln \rho_{n}(x)\right]^{2}\right\rangle^{-\frac{1}{2}}=\left\{\int_{\Delta} d x \frac{\left[\rho_{n}^{\prime}(x)\right]^{2}}{\rho_{n}(x)}\right\}^{-\frac{1}{2}},
$$

where $F\left[\rho_{n}\right] \equiv F\left[p_{n}\right]$ denotes the Fisher information of the polynomial $p_{n}(x)$. This quantity was derived for the first time from the second-order differential equation of HOPS [60] (see also $[25,61])$, obtaining the following values:

$$
(\delta x)_{n}=\frac{1}{\sqrt{4 n+2}},
$$

for Hermite polynomials $H_{n}(x)$,

$$
(\delta x)_{n, \alpha}=\left\{\begin{array}{cc}
\frac{1}{\sqrt{4 n+1}} ; & \alpha=0, \\
\sqrt{\frac{\alpha^{2}-1}{(2 n+1) \alpha+1} ;} & \alpha>1, \\
0 ; & \alpha \in(-1,+1], \alpha \neq 0 .
\end{array}\right.
$$

for Laguerre polynomials $L_{n}^{(\alpha)}(x), \alpha>-1$, and

$$
(\delta x)_{n, \alpha, \beta}=\frac{1}{\sqrt{F\left[\rho_{n, \alpha, \beta}\right]}},
$$


where the Fisher information $F\left[\rho_{n, \alpha, \beta}\right]$ is given by the following:

$$
F\left[\rho_{n, \alpha, \beta}\right]= \begin{cases}2 n(n+1)(2 n+1), & \alpha, \beta=0, \\ \frac{2 n+\beta+1}{4}\left[\frac{n^{2}}{\beta+1}+n+(4 n+1)(n+\beta+1)+\frac{(n+1)^{2}}{\beta-1}\right], & \alpha=0, \beta>1, \\ \frac{2 n+\alpha+\beta+1}{4(n+\alpha+\beta-1)}\left[n(n+\alpha+\beta-1)\left(\frac{n+\alpha}{\beta+1}+2+\frac{n+\beta}{\alpha+1}\right)\right. & \\ \left.+(n+1)(n+\alpha+\beta)\left(\frac{n+\alpha}{\beta-1}+2+\frac{n+\beta}{\alpha-1}\right)\right], & \alpha, \beta>1, \\ \infty, & \text { otherwise }\end{cases}
$$

for Jacobi polynomials $P_{n}^{(\alpha, \beta)}(x)$, with $\alpha, \beta>-1$. We can easily note from these values and the ones for the standard deviation of the previous section that the Cramér-Rao inequality (9) is duly fulfilled for the three families of HOPs. Moreover, we observe that the asymptotical behavior of the Fisher spreading length $\delta(x)$ for the HOPs is $n^{-1 / 2}$ (Hermite), $n^{-1 / 2}$ (Laguerre) and $n^{-3 / 2}$ (Jacobi) when the weight's parameters are fixed.

\section{Rényi's Spreading Lengths and Weighted $\mathcal{L}_{q}$-Norms of HOPs}

In this section, we give the values of the Rényi spreading length (6) for the three canonical families of HOPs $\left\{p_{n}(x)\right\}$, defined by the expressions (10) and (11). These quantities, denoted as $\mathcal{L}_{q}^{R}\left[p_{n}\right]$ for convenience, are given by the corresponding quantities of the associated Rakhmanov density $\rho_{n}(x)$ given by (1):

$$
\mathcal{L}_{q}^{R}\left[\hat{p}_{n}\right]=e^{R_{q}\left[\hat{p}_{n}\right]}=\left(W_{q}\left[\hat{p}_{n}\right]\right)^{\frac{1}{1-q}}=\left\{\int_{\Lambda}\left[\rho_{n}(x)\right]^{q} d x\right\}^{\frac{1}{1-q}}=\left\{\int_{\Lambda}\left[\hat{p}_{n}^{2}(x) h(x)\right]^{q} d x\right\}^{\frac{1}{1-q}}, \quad q>0, q \neq 1,
$$

where the symbols $R_{q}\left[\hat{p}_{n}\right]$ and $W_{q}\left[\hat{p}_{n}\right]$ for the Rényi entropy and the weighted $\mathcal{L}_{q}$-norm of the orthonormal polynomials $\hat{p}_{n}(x)$ denote the $q$ th-order Rényi entropy $R_{q}\left[\rho_{n}\right]$ and the entropic moment $W_{q}\left[\rho_{n}\right]$ of $\rho_{n}(x)$, respectively, so that the following holds:

$$
R_{q}\left[\hat{p}_{n}\right]=\frac{1}{1-q} \ln \int_{\Lambda}\left[\hat{p}_{n}^{2}(x) h(x)\right]^{q} d x, \quad q>0, q \neq 1,
$$

and

$$
W_{q}\left[\hat{p}_{n}\right]=\int_{\Lambda}\left[\hat{p}_{n}^{2}(x) h(x)\right]^{q} d x, \quad q>0, q \neq 1,
$$

Then, we have in particular that the weighted $\mathcal{L}_{q}$-norm and the Rényi spreading length of the orthogonal (HOPs) and orthonormal polynomials are mutually related as follows:

$$
W_{q}\left[p_{n}\right]=\int_{\Lambda}\left[p_{n}^{2}(x) h(x)\right]^{q} d x=\kappa_{n}^{q} W_{q}\left[\hat{p}_{n}\right]
$$

and

$$
\mathcal{L}_{q}^{R}\left[p_{n}\right]=\left(W_{q}\left[p_{n}\right]\right)^{\frac{1}{1-q}}=\kappa_{n}^{\frac{q}{1-q}}\left(W_{q}\left[\hat{p}_{n}\right]\right)^{\frac{1}{1-q}} .
$$

The analytical determination of these norms has been a long standing problem in the theory of special functions and extremal polynomials itself since the times of Bernstein and Steklov (see [62-64]) and in the theory of trigonometric series [65]. More recently, these quantities were obtained by using either (a) the series expansion of the powers of the HOPs $p_{n}(x)$ by means of the combinatorial Bell polynomials [66], or (b) the SrivastavaNiukkanen's linearization method $[67,68]$ of the positive integer powers $\left[p_{n}(x)\right]^{2 q}$ of HOPs by means of the multivariate Lauricella function $F_{A}^{(2 q)}\left(\frac{1}{q}, \ldots, \frac{1}{q}\right)$ [69] (Hermite and La-

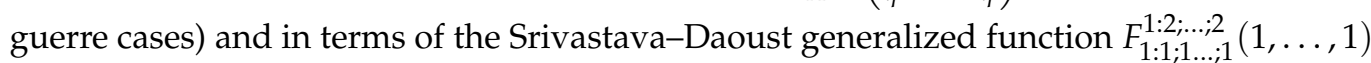
(Jacobi case), as Sánchez-Moreno et al. showed in [22,70], respectively. The combinatorial approach is based on the expansion $p$-th power of the arbitrary polynomial as follows:

$$
y_{n}(x)=\sum_{t=0}^{n} c_{t} x^{t}
$$


given by the following [22]:

$$
\left[y_{n}(x)\right]^{p}=\sum_{t=0}^{n p} \frac{p !}{(t+p) !} B_{t+p, p}\left(c_{0}, 2 ! c_{1}, \ldots,(t+1) ! c_{t}\right) x^{t},
$$

with $c_{i}=0$ for $i>n$; the remaining expansion coefficients are given by Equations (44), (49) and (56) in the Hermite, Laguerre and Jacobi cases, respectively. The $B$-symbols denote the renowned multivariate Bell polynomials of combinatorics [66] which are given by the following:

$$
B_{m, l}\left(c_{1}, c_{2}, \ldots, c_{m-l+1}\right)=\sum_{\hat{\pi}(m, l)} \frac{m !}{j_{1} ! j_{2} ! \cdots j_{m-l+1} !}\left(\frac{c_{1}}{1 !}\right)^{j_{1}}\left(\frac{c_{2}}{2 !}\right)^{j_{2}} \cdots\left(\frac{c_{m-l+1}}{(m-l+1) !}\right)^{j_{m-l+1}}
$$

where the sum runs over all partitions $\hat{\pi}(m, l)$ such that the following holds:

$$
j_{1}+j_{2}+\cdots+j_{m-l+1}=l, \quad \text { and } \quad j_{1}+2 j_{2}+\cdots+(m-l+1) j_{m-l+1}=m .
$$

On the other hand, the algebraic approach [70] uses the Srivastava-Niukanen linearization formulas of the HOPS. In particular, for the Laguerre polynomials we have the following [70]:

$$
x^{\mu}\left[L_{n}^{(\alpha)}(t x)\right]^{r}=\sum_{i=0}^{\infty} c_{i}(\mu, r, t, n, \alpha, \gamma) L_{i}^{(\gamma)}(x)
$$

with the following linearization coefficients:

$$
c_{i}(\mu, r, t, n, \alpha, \gamma)=(\gamma+1)_{\mu}\left(\begin{array}{c}
n+\alpha \\
n
\end{array}\right)^{r} F_{A}^{(r+1)}(\begin{array}{c}
\gamma+\mu+1 ; \overbrace{-n, \ldots,-n}^{r},-i \\
\underbrace{\alpha+1, \ldots, \alpha+1}_{r}, \gamma+1
\end{array} ; \overbrace{t, \ldots, t, 1}^{r}),
$$

where the Pochhammer symbol $(z)_{a}=\frac{\Gamma(z+a)}{\Gamma(z)}[6]$ and $F_{A}^{(r+1)}\left(x_{1}, \ldots, x_{r}\right)$ denote the Lauricella function of type A of $r+1$ variables and $2 r+3$ parameters defined as follows: [69]

$$
F_{A}^{(s)}\left(\begin{array}{c}
a ; b_{1}, \ldots, b_{s} \\
c_{1}, \ldots, c_{s}
\end{array} ; x_{1}, \ldots, x_{s}\right)=\sum_{j_{1}, \ldots, j_{s}=0}^{\infty} \frac{(a)_{j_{1}+\cdots+j_{s}}\left(b_{1}\right)_{j_{1}} \cdots\left(b_{s}\right)_{j_{s}}}{\left(c_{1}\right)_{j_{1}} \cdots\left(c_{s}\right)_{j_{s}}} \frac{x_{1}^{j_{1}} \cdots x_{s}^{j_{s}}}{j_{1} ! \cdots j_{s} !} .
$$

Similarly, we have the following linearization formula for the Jacobi polynomials [70]:

$$
\left(P_{n}^{(\alpha, \beta)}(x)\right)^{r}=\sum_{i=0}^{\infty} \tilde{c}_{i}(r, n, \alpha, \beta, \gamma, \delta) P_{i}^{(\gamma, \delta)}(x),
$$

where the coefficients are given by the following:

$$
\begin{aligned}
\tilde{c}_{i}(r, n, \alpha, \beta, \gamma, \delta)= & \left(\begin{array}{c}
n+\alpha \\
n
\end{array}\right)^{r} \frac{\gamma+\delta+2 i+1}{\gamma+\delta+i+1} \sum_{j_{1}, \ldots, j_{r}=0}^{n} \sum_{j_{r+1}=0}^{i} \frac{(\gamma+1)_{j_{1}+\cdots+j_{r}+j_{r+1}}}{(\gamma+\delta+i+2)_{j_{1}+\cdots+j_{r}}} \\
& \times \frac{(-n)_{j_{1}}(\alpha+\beta+n+1)_{j_{1}} \cdots(-n)_{j_{r}}(\alpha+\beta+n+1)_{j_{r}}(-i)_{j_{r+1}}}{(\alpha+1)_{j_{1}} \cdots(\alpha+1)_{j_{r}}(\gamma+1)_{j_{r+1}} j_{1} ! \cdots j_{r} ! j_{r+1} !},
\end{aligned}
$$

To obtain the linearization formula for the Hermite polynomials within the framework of this algebraic approach, we use Equation (36) together with the known relation of the Hermite and Laguerre polynomials [6]:

$$
H_{2 n}(x)=(-1)^{n} 2^{2 n} n ! L_{n}\left(x^{2}\right) ; \quad H_{2 n+1}(x)=(-1)^{n} 2^{2 n+1} n ! x L_{n}\left(x^{2}\right) .
$$




\subsection{Hermite Polynomials}

From (27) and the explicit expressions (32) of the Hermite polynomials $H_{n}(x)$, the combinatorial approach allows one to obtain the following values of the Rényi spreading lengths $\mathcal{L}_{q}^{R}\left[H_{n}\right]$ for $2 q \in \mathbb{N}, q>2$ :

$$
\mathcal{L}_{q}^{R}\left[H_{n}\right]=\left\{W_{q}\left[H_{n}\right]\right\}^{\frac{1}{1-q}},
$$

with the weighted norm $W_{q}\left[H_{n}\right]$ of the Hermite polynomials given by the following:

$$
W_{q}\left[H_{n}\right]=\int_{-\infty}^{\infty}\left[H_{n}^{2}(x) h_{H}(x)\right]^{q} d x=\sum_{j=0}^{n q} \frac{\Gamma\left(j+\frac{1}{2}\right)}{q^{j+\frac{1}{2}}} \frac{(2 q) !}{(2 j+2 q) !} B_{2 j+2 q, 2 q}\left(c_{0}^{(n)}, 2 ! c_{1}^{(n)}, \ldots,(2 j+1) ! c_{2 j}^{(n)}\right),
$$

with the following values:

$$
c_{t}^{(n)}=\frac{(-1)^{\frac{3 n-t}{2}} n !}{\left(2^{n} n ! \sqrt{\pi}\right)^{\frac{1}{2}}} \frac{2^{t}}{\left(\frac{n-t}{2}\right) ! t !} \frac{(-1)^{t}+(-1)^{n}}{2} .
$$

for the expansion coefficients $c_{t}$ of the orthonormal Hermite polynomials (see [3,6,22]). Then, the expressions (43) and (42) together with (34) and (44) provide an algorithmic procedure to find the $q$ th-weighted norm and the $q$ th-Rényi spreading length of the Hermite polynomial, respectively, in terms of its degree $n$ and the parameter $q$. See Section 3.2 of [22] for further details.

Alternatively, from Equations (36) and (41) and the orthogonality relation (10) of the Hermite polynomials, the algebraic approach [70] allows one to find the following values:

$$
W_{q}\left[H_{n}\right]=q^{-v q-\frac{1}{2}}\left(\frac{\Gamma\left(\frac{n+v+1}{2}\right)}{\Gamma\left(\frac{n-v}{2}+1\right)}\right)^{q}\left(\Gamma\left(v+\frac{1}{2}\right)\right)^{-2 q} \Gamma\left(q v+\frac{1}{2}\right) F_{A}^{(2 q)}\left(\begin{array}{c}
q v+\frac{1}{2} ; \overbrace{\frac{v-n}{2}, \ldots, \frac{v-n}{2}}^{2 q} \overbrace{\frac{1}{q}, \ldots, \frac{1}{q}}^{2 q} \\
\underbrace{v+\frac{1}{2}, \ldots, v+\frac{1}{2}}_{2 q}
\end{array}\right)
$$

for the weighted norm of Hermite polynomials. Note that this quantity is completely determined by $n$ and $q$. Let us recall here that $F_{A}^{(r+1)}\left(x_{1}, \ldots, x_{r}\right)$ denotes the Lauricella function of type A of $r+1$ variables and $2 r+3$ parameters defined as follows [69]:

$$
F_{A}^{(s)}\left(\begin{array}{c}
a ; b_{1}, \ldots, b_{s} \\
c_{1}, \ldots, c_{s}
\end{array} ; x_{1}, \ldots, x_{s}\right)=\sum_{j_{1}, \ldots, j_{s}=0}^{\infty} \frac{(a)_{j_{1}+\cdots+j_{s}}\left(b_{1}\right)_{j_{1}} \cdots\left(b_{s}\right)_{j_{s}}}{\left(c_{1}\right)_{j_{1}} \cdots\left(c_{s}\right)_{j_{s}}} \frac{x_{1}^{j_{1}} \cdots x_{s}^{j_{s}}}{j_{1} ! \cdots j_{s} !}
$$

which depends on $N=\sum_{i=0}^{r}\left(p_{i}+q_{i}\right)$ parameters.

Then, from expressions Equations (42), (43) and (45), one has the Rényi spreading length of the Hermite polynomials in the two combinatorial and algebraic approaches. In particular, for $q=2$, we obtain the following values for the Onicescu information-theoretic length $\mathcal{L}_{2}^{R}\left[H_{n}\right]=\mathcal{L}_{2}^{R}\left[\rho_{n}\right]$

$$
\mathcal{L}_{2}^{R}\left[H_{0}\right]=\sqrt{2 \pi}, \quad \mathcal{L}_{2}^{R}\left[H_{1}\right]=\frac{4}{3} \sqrt{2 \pi}, \quad \mathcal{L}_{2}^{R}\left[H_{2}\right]=\frac{64}{41} \sqrt{2 \pi}
$$

of the first few Hermite polynomials with degrees $n=0,1,2$. See $[22,70,71]$ for further details and application to the determination of the total Rényi entropies of the one- and multidimensional harmonic systems in position and momentum spaces. 


\subsection{Laguerre Polynomials}

From (27) and the explicit expressions (32) of the orthonormal Laguerre polynomials $\hat{L}_{n}^{(\alpha)}(x)$, the combinatorial approach allows one to obtain the following values of the Rényi spreading lengths $\mathcal{L}_{q}^{R}\left[\hat{L}_{n}^{(\alpha)}\right]=\mathcal{L}_{q}^{R}\left[\rho_{n, \alpha}\right]$ for $2 q \in \mathbb{N}, q>2$ and being $\rho_{n, \alpha}=\hat{L}_{n}^{(\alpha)}(x)^{2} h_{\alpha}^{L}(x)$ :

$$
\mathcal{L}_{q}^{R}\left[\hat{L}_{n}^{(\alpha)}\right]=\left\{W_{q}\left[\hat{L}_{n}^{(\alpha)}\right]\right\}^{\frac{1}{1-q}},
$$

with the weighted norm $W_{q}\left[L_{n}^{(\alpha)}\right]$ of the Laguerre polynomials given by the following:

$$
W_{q}\left[\hat{L}_{n}^{(\alpha)}\right]=\int_{0}^{\infty}\left[\hat{L}_{n}^{(\alpha)}(x)^{2} h_{\alpha}^{L}(x)\right]^{q} d x=\sum_{k=0}^{2 n q} \frac{\Gamma(\alpha q+k+1)}{q^{\alpha q+k+1}} \frac{(2 q) !}{(k+2 q) !} B_{k+2 q, 2 q}\left(c_{0}^{(n, \alpha)}, 2 ! c_{1}^{(n, \alpha)}, \ldots,(k+1) ! c_{k}^{(n, \alpha)}\right),
$$

where $B$-symbol describes the multivariate Bell polynomials to be calculated at the following values:

$$
c_{t}^{(n, \alpha)}=\sqrt{\frac{\Gamma(n+\alpha+1)}{n !}} \frac{(-1)^{t}}{\Gamma(\alpha+t+1)}\left(\begin{array}{l}
n \\
t
\end{array}\right) .
$$

for the expansion coefficients of the orthonormal Laguerre polynomials (see [6,24]). Then, the expressions (48) and (47) together with (34) and (49) provide an algorithmic procedure to find the $q$ th-weighted norm and the $q$ th-Rényi spreading length of the Laguerre polynomial, respectively, in terms of its degree $n$ and the parameters $\alpha$ and $q$. In particular, for $q=2$, we obtain the following values for the Onicescu information-theoretic length $\mathcal{L}_{2}^{R}\left[\hat{L}_{n}^{(\alpha)}\right]$ :

$$
\mathcal{L}_{2}^{R}\left[\hat{L}_{0}^{(\alpha)}\right]=\left(\frac{2^{2 \alpha+1}(\Gamma(\alpha+1))^{2}}{\Gamma(2 \alpha+1)}\right)^{\frac{1}{2}}, \quad \mathcal{L}_{2}^{R}\left[\hat{L}_{1}^{(\alpha)}\right]=\left(\frac{2^{2 \alpha+3}(\Gamma(\alpha+2))^{2}}{(1+\alpha)(2+3 \alpha) \Gamma(2 \alpha+1)}\right)^{\frac{1}{2}}
$$

of the Laguerre polynomials $L_{n}^{(\alpha)}(x)$ with $n=0,1$.

Alternatively, from the expression (36) and the orthogonality relation (10) of the Laguerre polynomials, the algebraic approach [70] allows one to find the following values:

$$
W_{q}^{R}\left[L_{n}^{(\alpha)}\right]=\frac{\Gamma(\alpha q+1)}{q^{\alpha q+1}} \frac{(\Gamma(n+\alpha+1))^{q}}{(n !)^{q}(\Gamma(\alpha+1))^{2 q}} F_{A}^{(2 q)}\left(\begin{array}{c}
\alpha q+1 ; \overbrace{-n, \ldots,-n}^{2 q} \overbrace{\frac{1}{2 q}, \ldots, \frac{1}{q}}^{2 q} \\
\underbrace{\alpha+1, \ldots, \alpha+1}_{2 q}
\end{array}\right),
$$

for the weighted norm of the Laguerre polynomials $L_{n}^{(\alpha)}(x)$ for all integer orders (i.e., when $q \in \mathbb{N}$ ). Note that these quantities only depend on the order $q$, the polynomial degree $n$ and the polynomial parameter $\alpha$, where $F_{A}^{(2 q)}\left(x_{1}, \ldots, x_{r}\right)$ denotes the Lauricella function of type A given by Equation (46).

Then, from expressions Equations (47) and (51) one has the Rényi spreading length of the Laguerre polynomials in the two combinatorial and algebraic approaches. In particular, we have the following values:

$$
\mathcal{L}_{q}^{R}\left[\hat{L}_{0}^{(\alpha)}\right]=\left[\frac{1}{\Gamma(\alpha+1)^{q}} \frac{\Gamma(\alpha q+1)}{q^{\alpha q+1}}\right]^{\frac{1}{1-q}}
$$

which recalls the Mehta-Selberg integral, and

$$
\mathcal{L}_{q}^{R}\left[\hat{L}_{1}^{(\alpha)}\right]=\left[\frac{\Gamma(\alpha q+1)(1+\alpha)^{2 q}}{(\Gamma(\alpha+2))^{q} q^{\alpha q+1}}{ }_{2} F_{0}\left(\begin{array}{c}
-2 q, \alpha q+1 \\
-
\end{array} ; \frac{1}{q(\alpha+1)}\right)\right]^{\frac{1}{1-q}} .
$$


for the Laguerre polynomials $L_{n}^{(\alpha)}(x)$ with $n=0,1$. Note also that Equations (52) and (53) with $q=2$ boil down to the previous values (50), respectively, of the Onicescu lengths. See $[70,72]$ for further details and application to the determination of the radial Rényi entropies of the multidimensional hydrogenic systems in position and momentum spaces.

\subsection{Jacobi Polynomials}

From (27) and the explicit expressions (32) of the Jacobi polynomials $P_{n}^{(\alpha, \beta)}(x)$, the combinatorial approach allows one to obtain the following values of the Rényi spreading lengths $\mathcal{L}_{q}^{R}\left[P_{n}^{(\alpha, \beta)}\right]=\mathcal{L}_{q}^{R}\left[\rho_{n, \alpha, \beta)}\right]$ for $2 q \in \mathbb{N}, q>2$ :

$$
\mathcal{L}_{q}^{R}\left[P_{n}^{(\alpha, \beta)}\right]=\left\{W_{q}\left[P_{n}^{(\alpha, \beta)}\right]\right\}^{\frac{1}{1-q}},
$$

with the weighted norm $W_{q}\left[P_{n}^{(\alpha, \beta)}\right]$ of the Jacobi polynomials given by the following:

$W_{q}\left[P_{n}^{(\alpha, \beta)}\right]=\int_{-1}^{+1}\left[P_{n}^{(\alpha, \beta)}(x)^{2} h_{\alpha, \beta}^{J}(x)\right]^{q} d x=\sum_{k=0}^{2 n q} \frac{(2 q) !}{(k+2 q) !} B_{k+2 q, 2 q}\left(c_{0}^{(n, \alpha, \beta)}, 2 ! c_{1}^{(n, \alpha, \beta)}, \ldots,(k+1) ! c_{k}^{(n, \alpha, \beta)}\right) \mathcal{I}(k, q, \alpha, \beta)$,

with the parametric function

$$
\mathcal{I}(k, q, \alpha, \beta)=\frac{(-1)^{k} 2^{1+\alpha q+\beta q} \Gamma(\alpha q+1) \Gamma(\beta q+1)}{\Gamma(\alpha q+\beta q+2)}{ }_{2} F_{1}\left(\begin{array}{c}
-k, 1+\beta q \\
2+(\alpha+\beta) q
\end{array} ; 2\right)
$$

with the Jacobi expansion coefficients (see $[3,6,23]$ )

$$
c_{t}^{(n, \alpha, \beta)}=\quad \sqrt{\frac{\Gamma(\alpha+n+1)(2 n+\alpha+\beta+1)}{n ! 2(\alpha+\beta+1) \Gamma(\alpha+\beta+n+1) \Gamma(n+\beta+1)}} \sum_{i=t}^{n}(-1)^{i-t}\left(\begin{array}{l}
n \\
i
\end{array}\right)\left(\begin{array}{c}
i \\
t
\end{array}\right) \frac{\Gamma(\alpha+\beta+n+i+1)}{2^{i} \Gamma(\alpha+i+1)}
$$

Then, the expressions (55) and (54) together with (34) and (56) provide an algorithmic procedure to find the $q$ th-weighted norm and the $q$ th-Rényi spreading length of the Jacobi polynomial, respectively, in terms of its degree $n$ and the parameters $(\alpha, \beta)$ and $q$. See Section 4 of [23] for further details.

Alternatively, from the expression (39) and the orthogonality relation (10) of the Jacobi polynomials, the algebraic approach [70] allows one to find the following values:

$$
\begin{aligned}
W_{q}\left[P_{n}^{(\alpha, \beta)}\right]=\left[\frac{2^{\alpha q+\beta q+1} \Gamma(\alpha q+1) \Gamma(\beta q+1)}{\Gamma(\alpha q+\beta q+2)}\left(\frac{n !(\alpha+\beta+2 n+1) \Gamma(\alpha+\beta+n+1)}{2^{\alpha+\beta+1} \Gamma(\alpha+n+1) \Gamma(\beta+n+1)}\right)^{q}\right. \\
\left.\quad \times\left(\begin{array}{c}
n+\alpha \\
n
\end{array}\right)^{2 q} F_{1: 1 ; \ldots ; 1}^{1: \ldots ; 2}\left(\begin{array}{c}
\alpha q+1: \overbrace{-n, \alpha+\beta+n+1 ; \ldots ;-n, \alpha+\beta+n+1}^{2 q} ; \overbrace{1, \ldots, 1}^{2 q} \\
\alpha q+\beta q+2: \underbrace{\alpha+1 ; \ldots ; \alpha+1}_{2 q}
\end{array}\right)\right]
\end{aligned}
$$

for the weighted norms of the Jacobi polynomials $P_{n}^{(\alpha, \beta)}(x)$ with order $q=2,3,4, \ldots$. Note that these quantities depend only on the order $q$, the polynomial degree $n$ and parameters $\alpha$ and $\beta$. Let us recall here that the symbol $F_{1: 1 ; \ldots ; 1}^{1: 2 ; \ldots ; 2}\left(x_{1}, \ldots, x_{r}\right)$ denotes the $r$-variate SrivastavaDaoust function defined as follows $[67,70]$ : 


$$
\begin{array}{r}
F_{1: 1 ; \ldots ; 1}^{1: 2 ; \ldots ; 2}\left(\begin{array}{c}
a_{0}^{(1)}: a_{1}^{(1)}, a_{1}^{(2)} ; \ldots ; a_{r}^{(1)}, a_{r}^{(2)} \\
b_{0}^{(1)}: b_{1}^{(1)}, \ldots ; b_{r}^{(1)}
\end{array}\right)= \\
=\sum_{j_{1}, \ldots, j_{r}=0}^{\infty} \frac{\left(a_{0}^{(1)}\right)_{j_{1}+\ldots+j_{r}} \frac{\left(a_{1}^{(1)}\right)_{j_{1}}\left(a_{1}^{(2)}\right)_{j_{1}} \cdots\left(a_{r}^{(1)}\right)_{j_{r}}\left(a_{r}^{(2)}\right)_{j_{r}}}{\left(b_{0}^{(1)}\right)_{j_{1}+\ldots+j_{r}}} \frac{x_{1}^{j_{1}} x_{2}^{j_{2}} \ldots x_{r}^{j_{r}}}{j_{1} ! j_{2} ! \cdots j_{r} !}}{\left(b_{1}^{(1)}\right)_{j_{1}}\left(b_{r}^{(1)}\right)_{j_{r}}}
\end{array}
$$

which depends on $N=\sum_{i=0}^{r}\left(p_{i}+q_{i}\right)$ parameters.

Then, from the expressions of Equations (54), (55) and (57), one has the Rényi spreading length of the Laguerre polynomials in the two combinatorial and algebraic approaches [23]. See $[67,70,72]$ for further details and application to the determination of the angular Rényi entropies of the multidimensional hydrogenic systems in position and momentum spaces.

Finally, since the expressions obtained for the weighted norms $W\left[p_{n}\right]$ and, consequently, for the Rényi entropies $R\left[p_{n}\right]$ and the Rényi spreading lengths $\mathcal{L}_{1}^{R}\left[p_{n}\right]$ of the HOPs are not easily manipulated analytically, it would be interesting at least to have the asymptotical values $(n \rightarrow \infty)$ and to obtain simple, compact and accurate upper bounds to these quantities in terms of the degree and the parameters of their weight function. The latter problem has not yet been determined, but the former one related to the asymptotics $(n \rightarrow \infty)$ is extensively shown in the next three sections for the Hermite, Laguerre and Jacobi polynomials and its generalizations.

\section{Degree Asymptotics for the Weighted $\mathfrak{L}_{q}$-Norms of Hermite Polynomials}

The aim of this section is the strong asymptotic $(n \rightarrow \infty)$ determination of the entropic moments or weighted $\mathfrak{L}_{q}$-norms of Hermite polynomials, i.e., the following:

$$
W_{q}\left[H_{n}\right]=\int_{-\infty}^{+\infty}\left[H_{n}^{2}(x) e^{-x^{2}}\right]^{q} d x ; \quad q>0 .
$$

This problem was initiated by Aptekarev et al. [34,36] who solved it for $q \in\left[0, \frac{4}{3}\right]$ by use of an extension of the Plancherel and Rotach asymptotics of the orthonormal Hermite polynomials [1]. They obtained [34] the following expression:

$$
\int_{-\infty}^{+\infty}\left[\hat{H}_{n-1}^{2}(x) e^{-x^{2}}\right]^{q} d x=c_{q}(2 n)^{\frac{1-q}{2}}(1+o(1)), \quad \text { for } q \leq \frac{4}{3},
$$

for the main term of the weighted norm of the orthonormal polynomials $\tilde{H}_{n-1}(x)$, with the following constant:

$$
c_{q}=\left(\frac{2}{\pi}\right)^{q} \frac{\Gamma\left(q+\frac{1}{2}\right)}{\Gamma(q+1)} \frac{\Gamma\left(1-\frac{q}{2}\right)}{\Gamma\left(\frac{3}{2}-\frac{q}{2}\right)} .
$$

Now, taking into account the normalization constant (12) and the Stirling formula for the gamma function [6], this expression gives rise to the following asymptotics for the weighted norm of the orthogonal Hermite polynomial $H_{n-1}(x)$ :

$$
W_{q}\left[H_{n-1}\right]=c_{q} \kappa_{n-1}^{q}(2 n)^{\frac{1-q}{2}}(1+o(1))=c_{q} \pi^{q}(2 n)^{q(n-1)+1 / 2} e^{-q n}(1+o(1)) .
$$

To improve this expression and extends its validity for all values of $q$, we have to go beyond the Plancherel-Rotach asymptotics since it cannot give the asymptotics of the Hermite polynomials on the whole real line. This can be achieved either by means of the powerful Riemann-Hilbert method of Deift et al. [73] (see also [74]) or by the Tulyakov approach [75], whose starting point is the weight of orthogonality and the recurrence relation, which characterizes these orthogonal polynomials, respectively. The application of the latter approach to Hermite polynomials has allowed us to find [76] the following asymptotical result: 
Theorem 1. Let $H_{n}(x)$ be the Hermite polynomials with the standard orthogonality (10)-(12). Then, the entropic moments or weighted $\mathfrak{L}_{q}$-norms $W_{q}\left[\rho_{n-1}\right]$, given by Equation (59), have the following asymptotic $(n \rightarrow \infty)$ behavior:

$$
W_{q}\left[H_{n-1}\right]= \begin{cases}c_{q} \pi^{q}(2 n)^{q(n-1)+1 / 2} e^{-q n}(1+o(1)), & q<2, \\ 2(2 n)^{2 n-\frac{3}{2}} e^{-2 n}(\ln (n)+O(1)), & q=2, \\ 2 C_{q} 2^{-q}(2 n)^{q\left(n-\frac{2}{3}\right)-\frac{1}{6}} e^{-q n}(1+o(1)), & q>2 .\end{cases}
$$

where the constant $c_{q}$ is defined in (61) and the constant $C_{q}$ is equal to the following:

$$
C_{q}=\int_{-\infty}^{+\infty}\left[\frac{2 \pi}{\sqrt[3]{2}} \operatorname{Ai}^{2}\left(-\frac{z \sqrt[3]{2}}{2}\right)\right]^{q} d z
$$

with $z:=\frac{2 n}{x^{\frac{2}{3}}}-x^{\frac{4}{3}}$, and the Airy function Ai (see [6]).

We note that the first asymptotic formula in the right hand side of (63) coincides with (62), but now it holds true in the maximal range of $p$ (when $p=2$, then $c_{p}=\infty$ ); let us also highlight that the main term of the asymptotics is growing. Moreover, the smaller terms contain a constant which depends on $p$, and when $p \rightarrow 0$, this constant tends to infinity; however, our formula is correct for any small fixed $p>0$. We also note that the leading term of all three formulae in the right hand side of (63) match each other when $p \rightarrow 2$.

Let us highlight the great simplicity of the main term of asymptotics $(n \rightarrow \infty)$ of the weighted norm of the Hermite polynomials, $W_{q}\left[H_{n}\right]$, given by expressions (63). Indeed, this result is impressively simple, given the complexity of the expressions (43) and (45) obtained in Section 5 for the corresponding quantities of a Hermite polynomial with fixed degree $n$, which require the evaluation of either the multivariate Bell polynomials (34) at the Hermite expansion coefficients (44) or the multivariate Lauricella function of type A of the type (46) at $1 / q$, respectively.

This theorem facilitates the evaluation of the Rényi entropies of the highly-excited (i.e., Rydberg) states of the one-dimensional oscillator-like systems [76], basically because the radial wave functions of these quantum systems are controlled by Hermite's polynomials. Briefly, the various physical quantities described by the Rényi entropies with different orders $q$ have a clearly increasing dependence on the principal quantum number $n$ characterizing the Rydberg state of the system.

\section{Degree Asymptotics for the Generalized Weighted $\mathfrak{L}_{q}$-Norms of Laguerre Polynomials}

In this section, we study and solve the asymptotics $(n \rightarrow \infty)$ of the generalized weighted $\mathfrak{L}_{q}$-norms of the Laguerre polynomials defined as follows:

$$
N_{n}^{(q)}(\alpha, \beta)=\int_{0}^{\infty}\left(\left[\widehat{L}_{n}^{(\alpha)}(x)\right]^{2} h_{\alpha}^{L}(x)\right)^{q} x^{\beta} d x, \quad q>0, \alpha>-1 .
$$

with $\beta$ so that $\beta+p \alpha>-1$ to get convergence, and the weight function $h_{\alpha}^{L}(x)=x^{\alpha} e^{-x}$. Note that for $\beta=0$ these quantities denote the weighted $\mathfrak{L}_{q}$-norms (29) of the orthonormal Laguerre polynomials, $W_{q}\left[\widehat{L}_{n}^{(\alpha)}\right]$. The orthonormal polynomials $\left[\widehat{L}_{n}^{(\alpha)}(x)\right]^{2}=\left[L_{n}^{(\alpha)}(x)\right]^{2} / \kappa_{n}^{L}$, with the norm $\kappa_{n}^{L}=\left\|L_{n}^{(\alpha)}\right\|^{2}=d_{n, \alpha}^{2}$ given by (13) and the orthogonal polynomials:

$$
L_{n}^{(\alpha)}(x)=\sum_{\nu=0}^{n}\left(\begin{array}{l}
n+\alpha \\
n-v
\end{array}\right) \frac{(-x)^{v}}{v !} .
$$


This problem, which essentially depends on the values of the parameters $\alpha, \beta$ and $q$, was tackled by Aptekarev et al. [77] in 2016. They realized that the dominant contribution to the integral $N_{n}^{(q)}(\alpha, \beta)$ is given by different regions of integration in (64) depending on the parameters' values, which control the behavior of the polynomials along its orthogonality interval. Accordingly, we have to use different asymptotical representations for the Laguerre polynomials in the various integration's regions of the orthogonality interval $(0, \infty)$; these representations appear to follow different scales.

First, at the extreme left of the orthogonality interval (i.e., in the neighborhood of zero) we have the Bessel regime because the Laguerre polynomials can be asymptotically represented by means of Bessel functions. Then, to the right, in the bulk region of zeros' location, the oscillatory behavior of the polynomials is modeled asymptotically by means of the trigonometric functions. At the neighborhood of the extreme right zeros, asymptotics of the polynomials is given by Airy functions: it is the Airy regime. Finally, in the neighborhood of $\infty$ the polynomials have growing asymptotics. Furthermore, in some contiguous integration's regions, the asymptotical behaviors appear to match each other. Namely, asymptotics of the Bessel functions for large arguments match the trigonometric function, and asymptotics of the Airy functions do the same.

There are in total five asymptotical regimes that can give (depending on $\alpha$ and $q$ ) the dominant contribution in the asymptotics of $N_{n}^{(q)}(\alpha, \beta)$. Three of them show a growing dependence on $n$, according to a power law with an exponent, which depends on $\alpha$ and $q$; we refer to them as Bessel, Airy and cosine (or oscillatory) regimes. In addition, there are two more asymptotical regimes associated to the transition regions: cosine-Bessel and cosine-Airy. If these regimes dominate in integral (64), then the asymptotics of $N_{n}^{(q)}(\alpha, \beta)$ has a factor $\ln n$ besides the power law in $n$. Summarizing, a detailed and highbrow analysis led Aptekarev et al. [77] to the following result.

Theorem 2. The asymptotics $(n \rightarrow \infty)$ of the generalized weighted $\mathfrak{L}_{q}$-norms $N_{n}^{(q)}(\alpha, \beta)$ of the Laguerre polynomials defined by (64), is given by the following expressions:

A. For $\beta>0$,

$$
N_{n}^{(q)}(\alpha, \beta)= \begin{cases}C(\beta, q)(2 n)^{1+\beta-q}(1+o(1)), & q \in(0,2) \\ \frac{\ln n+O(1)}{\pi^{2}(4 n)^{1-\beta}}, & q=2 \\ \frac{C_{A}(q)}{\pi^{q}}(4 n)^{\left(\frac{1-2 q}{3}+\beta\right)}(1+o(1)), & q \in(2,2+3 \beta), \\ \left(\frac{C_{A}(q)}{\pi^{q} 4^{\beta+1}}+C_{B}(\alpha, \beta, q)\right) n^{-\beta-1}, & q=2+3 \beta \\ C_{B}(\alpha, \beta, q) n^{-\beta-1}, & q \in(2+3 \beta, \infty)\end{cases}
$$

B. For $\beta=0$,

$$
N_{n}^{(q)}(\alpha, 0) \equiv W_{q}\left[\widehat{L}_{n}^{(\alpha)}\right]= \begin{cases}C(0, q)(2 n)^{(1-q)}(1+o(1)), & q \in(0,2) \\ \frac{\ln n+O(1)}{\pi^{2} n}, & q=2 \\ \frac{C_{B}(\alpha, 0, q)}{n}(1+o(1)), & q>2\end{cases}
$$


C. For $\beta<0$ (and $p>0$ ),

$$
N_{n}^{(q)}(\alpha, \beta)=\left\{\begin{array}{ll}
C(\beta, q)(2 n)^{1+\beta-q}(1+o(1)), & q \in(0,2+2 \beta) \\
\frac{2 \Gamma(q+1 / 2)(\ln n+O(1))}{\pi^{q+1 / 2} \Gamma(q+1)(4 n)^{1+\beta}}, & q=2+2 \beta \\
C_{B}(\alpha, \beta, q) n^{-(1+\beta)}(1+o(1)), & q>2+2 \beta
\end{array},\right.
$$

respectively, where the characteristics constants $C, C_{B}, C_{A}$ are given by the following:

$$
C_{B}(\alpha, \beta, q):=2 \int_{0}^{\infty} t^{2 \beta+1}\left|J_{\alpha}(2 t)\right|^{2 q} d t .
$$

for the Bessel regime,

$$
C_{A}(q):=\int_{-\infty}^{+\infty}\left[\frac{2 \pi}{\sqrt[3]{2}} \operatorname{Ai}^{2}\left(-\frac{t \sqrt[3]{2}}{2}\right)\right]^{q} d t
$$

for the Airy regime, and

$$
C(\beta, q):=\frac{2^{\beta+1}}{\pi^{q+1 / 2}} \frac{\Gamma(\beta+1-q / 2) \Gamma(1-q / 2) \Gamma(q+1 / 2)}{\Gamma(\beta+2-q) \Gamma(1+q)} .
$$

for the cosine regime, respectively. The symbols $J_{\alpha}(z)$ and $A i(-z)$ denote the known Bessel and Airy functions [6,77], respectively.

Note that in all cases, the great simplicity of the main term of asymptotics $(n \rightarrow \infty)$ for the the generalized weighted $\mathfrak{L}_{q}$-norms $N_{n}^{(q)}(\alpha, \beta)$. This is more impressive for the weighted $\mathfrak{L}_{q}$-norms of the Laguerre polynomials, $N_{n}^{(q)}(\alpha, 0)=W_{q}\left[\widehat{L}_{n}^{(\alpha)}\right]$, given by expressions (67). Indeed, this result is amazingly simple, given the complexity of the expressions (48) and (51) obtained in Section 5 for the corresponding quantities of a Laguerre polynomial with arbitrary, fixed degree $n$, which require the evaluation of either the multivariate Bell polynomials (34) at the Laguerre expansion coefficients (49), or the multivariate Lauricella function of type A of the type (46) at $1 / q$, respectively.

These simple, compact asymptotical results were used to calculate the Rényi and Shannon entropies of the highly-excited (Rydberg) states of the three and multidimensional harmonic [77] and hydrogenic [40] systems. This is basically because the radial wave functions of these quantum systems are controlled by Laguerre's polynomials.

\section{Degree Asymptotics for Generalized Weighted $\mathfrak{L}_{q}$-Norms of OPs with General and Jacobi Weights}

In this section, we investigate and solve the asymptotics of the generalized weighted $\mathfrak{L}_{q}$-norms $N_{n}^{(q)}(h, F)$ of the polynomials $\hat{p}_{n}(x)$ orthonormal with respect to a general weight function (defined almost everywhere on $[-1,1]$ ) given by the following:

$$
N_{n}^{(q)}(h, F):=\int_{-1}^{1}\left[\hat{p}_{n}^{2}(x) h(x)\right]^{q} F(x) d x
$$

with

$$
F(x):=(1-x)^{\alpha}(1+x)^{\beta} f(x), \quad \alpha \geq \beta>-1, \quad f(x) \in \mathfrak{L}_{1}(-1,1),
$$

Then, we use this general asymptotics result to obtain the generalized weighted $\mathfrak{L}_{q}$-norms of the Jacobi polynomials $P_{n}^{(\alpha, \beta)}(x)$ and, as instances, the (simple) weighted $\mathfrak{L}_{q}$-norms denoted by $W_{q}\left[P_{n}^{(\alpha, \beta)}\right]$. 
Let us begin by considering the sequence $\left\{\hat{p}_{n}(x)\right\}_{n=0}^{\infty}$ of the orthonormal polynomials as follows:

$$
\int_{-1}^{1} \hat{p}_{n}(x) \hat{p}_{m}(x) h(x) d x=\delta_{n, m}, \quad \operatorname{deg} p_{n}=n
$$

with respect to a general weight function

$$
h(x)>0, \quad \text { a.e. } \quad x \in[-1,1] .
$$

To obtain the dominant term in the asymptotics of the norms $N_{n}^{(q)}(h, F)$, we first realize that the weight function $h(x)$ in (74) satisfies the Szegö condition as follows:

$$
\int_{-1}^{1} \frac{h(x)}{\sqrt{1-x^{2}}} d x>-\infty
$$

(which is more restrictive than (75)), then the orthonormal polynomials are known to have [1] the following asymptotical behavior on $[-1,1]$ :

$$
\int_{-1}^{1}\left|\hat{p}_{n}(x)-\bar{p}_{n}(x)\right|^{2} h(x) d x \rightarrow 0, \quad \text { when } n \rightarrow \infty,
$$

where the polynomials

$$
\bar{p}_{n}(x):=\sqrt{\frac{2}{h_{0}}} \cos (n \arccos x+\bar{\gamma}(x))
$$

form a set of polynomials orthonormal with respect to the weight $h_{0}(x):=\pi \sqrt{1-x^{2}} h(x)$, and where $\bar{\gamma}(x)$ is the Hilbert transform of the function $\ln h_{0}(x)$. Second, we take into account the following Lemma [34].

Lemma 1. Let $g$ be a continuous, periodic function on $\mathbb{R}, f$ be an integrable and $\tilde{\gamma}$ be a measurable function on $(0, \pi)$ :

$$
g \in C(\mathbb{R}), \quad g(\theta+\pi)=g(\theta), \quad f \in L^{1}(0, \pi), \quad \tilde{\gamma}<\text { const a.e. on }(0, \pi) .
$$

Then, the following holds:

$$
\int_{0}^{\pi} g(n \theta+\tilde{\gamma}(\theta)) f(\theta) d \theta \rightarrow \frac{1}{\pi} \int_{0}^{\pi} g(\theta) d \theta \int_{0}^{\pi} f(\theta) d \theta, \quad n \rightarrow \infty .
$$

Now, with these two previous results, Aptekarev et al. [78] proved in 2021 the following powerful result.

Theorem 3. Let $\left\{\hat{p}_{n}(x)\right\}$ be the polynomials (74) orthonormal on $[-1,1]$ with respect to the weight function $h(x)$ for which (a) has singularities at the end points \pm 1 of the order $(1-x)^{\alpha_{h}}$ and $(1+x)^{\beta_{h}}$, respectively, with $\alpha_{h} \geq \beta_{h}>-1$, and (b) satisfies the Szegö condition (76). Let also the following hold:

$$
F(x):=(1-x)^{\alpha}(1+x)^{\beta} f(x), \quad \alpha \geq \beta>-1, \quad f(x) \in \mathfrak{L}_{1}(-1,1),
$$

where $f(x)$ may have a possible growth at the end points \pm 1 but not faster than logarithmic. Then, we have the following asymptotical behavior: 


$$
N_{n}^{(q)}(h, F):=\int_{-1}^{1}\left[\hat{p}_{n}^{2}(x) h(x)\right]^{q} F(x) d x \quad \underset{n \rightarrow \infty}{\longrightarrow} \frac{2^{q} \bar{\Gamma}(q)}{\pi^{q+1}} \int_{-1}^{1}\left(1-x^{2}\right)^{-\frac{q}{2}} F(x) d x
$$

for

$$
-\frac{\beta+1}{\beta_{h}}<q<\beta+1
$$

and where

$$
\bar{\Gamma}(q):=\frac{\Gamma(q+1 / 2) \Gamma(1 / 2)}{\Gamma(q+1)} .
$$

Moreover, when $h(x)$ and $F(x)$ are the Jacobi weights, we can find, using the methodology of [34], the following asymptotical result [78] in a straightforward manner.

Theorem 4. Let $\left\{\hat{p}_{n}(x)\right\}$ be a system of Jacobi polynomials, orthonormal with respect to an arbitrary Jacobi weight function $h(x):=(1-x)^{\alpha_{h}}(1+x)^{\beta_{h}}, \alpha_{h} \geq \beta_{h}>-1$, and $F(x)$ be the Jacobi weight:

$$
F(x):=(1-x)^{\alpha}(1+x)^{\beta}, \quad \alpha \geq \beta>-1 .
$$

Then, the generalized weighted norm of the Jacobi polynomials

$$
N_{n}^{(q)}(h, F):=\int_{-1}^{1}\left[\hat{p}_{n}^{2}(x) h(x)\right]^{q} F(x) d x
$$

has the following limiting $(n \rightarrow \infty)$ behavior.

A. For $-\frac{\beta+1}{\beta_{h}}<q<2(\beta+1)$

$$
\begin{aligned}
N_{n}^{(q)}(h, F) & \longrightarrow \frac{2^{q}}{\pi^{q+1}} \bar{\Gamma}(q) \int_{-1}^{1}\left(1-x^{2}\right)^{-\frac{q}{2}} F(x) d x= \\
& =\frac{2^{\alpha+\beta+1}}{\pi^{q+1}} \frac{\Gamma\left(q+\frac{1}{2}\right) \Gamma\left(\frac{1}{2}\right)}{\Gamma(q+1)} \frac{\Gamma\left(\alpha-\frac{q}{2}+1\right) \Gamma\left(\beta-\frac{q}{2}+1\right)}{\Gamma(\alpha+\beta-q+2)}
\end{aligned}
$$

B. For $q=2(\beta+1)$

$$
N_{n}^{(q)}(h, F) \sim \ln n, \quad \text { i.e. } \exists c_{0}: N_{n}^{(q)} / \ln n \rightarrow c_{0} ;
$$

C. For $q>2(\beta+1)$

$$
N_{n}^{(q)}(h, F) \asymp n^{q-2(\beta+1)}, \quad \text { i.e. } \exists c_{1}, c_{2}: c_{1} \leq N_{n}^{(q)} / n^{q-2(\beta+1)} \leq c_{2} .
$$

Note the diversity of the asymptotics of $N_{n}^{(q)}$ when $n \rightarrow \infty$ for various $q$. For $q$ relatively small to the parameter $\beta$, the integrals remain bounded for big $n$. When $q$ approaches its critical value $2(\beta+1)$, the right hand side of the bound (84) explodes since the argument of the multiplier $\Gamma\left(\beta-\frac{q}{2}+1\right)$ is close to the pole of gamma function. Then, for the critical $q=2(\beta+1)$, the bound (84) does not work any more and according to (85), a new asymptotical regime takes place where $N_{n}^{(q)}$ grows as $\ln n$. Finally, when $q>2(\beta+1)$, the norms shows a $n$-degree exponential scale governed by the positive difference between $q$ and its critical value.

It is remarkable the great simplicity of the main term of asymptotics $(n \rightarrow \infty)$ of the weighted $\mathfrak{L}_{q}$-norms of the Jacobi polynomials, $W_{q}\left[\hat{P}_{n}^{(\alpha, \beta)}\right]$, given as particular instances of the expressions (84)-(86). Indeed, this result is impressively simple, given the complexity of the expressions (55) and (57) obtained in Section 5 for the corresponding quantities of a 
Jacobi polynomial with fixed degree $n$, which require the evaluation of either the multivariate Bell polynomials (34) at the Jacobi expansion coefficients (56), or the multivariate Srivastava-Daoust of the type (58) at unity, respectively.

These mathematical results were recently used [78] to evaluate in a compact and elegant way the Rényi and Shannon entropies of the Rydberg hydrogenic three and multidimensional states. This was possible because the radial and angular wave functions of these quantum states are controlled by the Gegenbauer polynomials, which are instances of the great family of Jacobi polynomials.

\section{Shannon's Spreading Length and Logarithmic Potential of HOPs}

In this section, we examine the Shannon spreading length $\mathcal{L}_{1}^{\mathcal{S}}\left[p_{n}\right]=\mathcal{L}_{1}^{\mathcal{S}}\left[\rho_{n}\right]$ of the three canonical families of the HOPs $\left\{p_{n}(x)\right\}$ for all $n$ by means of the logarithmic-potentialbased approach, which is the only existing one with general validity, although it requires the evaluation of the logarithmic potentials of the polynomials at their zeros on the orthogonality support. The latter, however, is a non-trivial task which makes necessary, almost mandatory, the consideration and analysis of the asymptotics $(n \rightarrow \infty)$ for the Shannon entropy of the HOPs; this is shown in the next section. Likewise, it is helpful to obtain analytical upper bounds to $\mathcal{L}_{1}^{\mathcal{S}}\left[p_{n}\right]$ as simply and accurately as possible; some results, which are briefly pointed out at the end of this section, were found for Hermite, Laguerre and Jacobi polynomials [22-24] in terms of the ordinary moments of their associated Rakhmanov density (1).

According to Equations (7), (10) and (11), this quantity is given by the Shannon spreading length of the associated Rakhmanov probability density $\rho_{n}(x)$ given by (1), that is, the following:

$$
\mathcal{L}_{1}^{S}\left[\rho_{n}\right]=\lim _{q \rightarrow 1} \mathcal{L}_{q}^{R}\left[\rho_{n}\right]=\exp \left(S\left[\rho_{n}\right]\right)=\exp \left\{-\int_{\Delta} \rho_{n}(x) \ln \rho_{n}(x) d x\right\},
$$

where $S\left[\rho_{n}\right]$ denotes the Shannon-like integral functional $S\left[p_{n}\right]$ of the polynomials given by the following:

$$
S\left[p_{n}\right]=S\left[\rho_{n}\right]=-\int_{\Lambda} \frac{1}{\kappa_{n}} p_{n}^{2}(x) h(x) \ln \left[\frac{1}{\kappa_{n}} p_{n}^{2}(x) h(x)\right] d x=\ln \kappa_{n}+\frac{1}{\kappa_{n}}\left(E\left[p_{n}\right]+I\left[p_{n}\right]\right),
$$

with the polynomial functionals

$$
I\left[p_{n}\right]:=-\int_{\Lambda} p_{n}^{2}(x) h(x) \ln h(x) d x
$$

and

$$
E\left[p_{n}\right]:=-\int_{\Lambda} p_{n}^{2}(x) h(x) \ln p_{n}^{2}(x) d x .
$$

The functional $I\left[p_{n}\right]$ was determined [79] for all HOPs in terms of the coefficients of its second-order differential equation. However, the explicit computation of the functional $E\left[p_{n}\right]$ for all $n$ in terms of the degree and the parameters of the weight function $h(x)$ is a formidable task, not yet solved for the HOPs despite the efforts of many authors, except (a) for a few specific families of the Jacobi polynomials, such as the Chebyshev polynomials of the first and second type, and (b) in some asymptotical cases, such as when $(n \rightarrow \infty)$ or when the parameters of the weight $h(x)$ go toward $\infty$. This functional $E\left[p_{n}\right]$ is called Shannon entropy of the HOPs $p_{n}(x)$. Note that, to fix the notation, the Shannon-like integral functional $S\left[\hat{p}_{n}\right]$ of the orthonormal polynomial $\hat{p}_{n}$ is the following:

$$
S\left[\hat{p}_{n}\right]=-\int_{\Lambda} \hat{p}_{n}^{2}(x) h(x) \ln \left[\hat{p}_{n}^{2}(x) h(x)\right] d x=E\left[\hat{p}_{n}\right]+I\left[\hat{p}_{n}\right],
$$


and the Shannon entropy of $\hat{p}_{n}(x)$ is the following:

$$
E\left[\hat{p}_{n}\right]:=-\int_{\Lambda} \hat{p}_{n}^{2}(x) h(x) \ln \hat{p}_{n}^{2}(x) d x,
$$

Then, for illustration, the Shannon spreading length for the orthonormal Hermite polynomials $\hat{H}_{n}(x)$ is given by the following:

$$
\mathcal{L}_{1}^{S}\left[\hat{H}_{n}\right]=\exp \left\{S\left[\hat{H}_{n}\right]\right\}
$$

where the Shannon-like integral functional of $\hat{H}_{n}(x)$ is the following:

$$
S\left[\hat{H}_{n}\right]=-\int_{\Lambda} \hat{H}_{n}^{2}(x) h_{H}(x) \ln \left[\hat{H}_{n}^{2}(x) h_{H}(x)\right] d x=E\left[\hat{H}_{n}\right]+n+\frac{1}{2},
$$

where $E\left[\hat{H}_{n}\right]$ denotes the Shannon entropy of $\hat{H}_{n}(x)$ defined as follows:

$$
E\left[\hat{H}_{n}\right]:=-\int_{\Lambda} \hat{H}_{n}^{2}(x) h_{H}(x) \ln \hat{H}_{n}^{2}(x) d x,
$$

whose explicit value for all $n$ has not yet been found despite serious attempts $[10,34,80]$. Only the asymptotic case $(n \rightarrow \infty)$ has been determined in a simple compact way as discussed in the next section.

Now, the Shannon spreading length of the orthonormal Laguerre polynomials is given by the following:

$$
\mathcal{L}_{1}^{S}\left[\hat{L}_{n}^{(\alpha)}\right]=\exp \left\{S\left[\hat{L}_{n}^{(\alpha)}\right]\right\}
$$

where the Shanon-like integral functional of the orthonormal Laguerre polynomials $\hat{L}_{n}^{(\alpha}(x)$ is the following:

$$
S\left[\hat{L}_{n}^{(\alpha)}\right]=-\int_{-1}^{+1}\left[\hat{L}_{n}^{(\alpha)}(x)\right]^{2} h_{\alpha}^{L}(x) \ln \left\{\left[\hat{L}_{n}^{(\alpha)}(x)\right]^{2} h_{\alpha}^{L}(x)\right\} d x=E\left[\hat{L}_{n}^{(\alpha)}\right]+I\left[\hat{L}_{n}^{(\alpha)}\right]
$$

with the integral functional as follows [24,79]:

$$
I\left[\hat{L}_{n}^{(\alpha)}\right]=-\int_{0}^{\infty}\left[\hat{L}_{n}^{(\alpha)}(x)\right]^{2} h_{\alpha}^{L}(x) \ln h_{\alpha}^{L}(x)=2 n+\alpha+1-\alpha \psi(\alpha+n+1)
$$

and the Shannon entropy of $\hat{L}_{n}^{(\alpha}(x)$ is given by the following:

$$
E\left[\hat{L}_{n}^{(\alpha)}\right]=-\int_{0}^{\infty}\left[\hat{L}_{n}^{(\alpha)}(x)\right]^{2} h_{\alpha}^{L}(x) \ln \left[\hat{L}_{n}^{(\alpha)}(x)\right]^{2} d x
$$

whose explicit value for all $n$ has not yet been found in spite of different attempts $[10,34,80]$; only the asymptotic case $(n \rightarrow \infty)$ has been determined in a simple compact way as is discussed in the next section.

Finally, the Shannon spreading length of the orthonormal Jacobi polynomials $\hat{P}_{n}^{(\alpha, \beta)}(x)$, which is given by the following:

$$
\mathcal{L}_{1}^{S}\left[\hat{P}_{n}^{(\alpha, \beta)}\right]=\exp \left\{S\left[\hat{P}_{n}^{(\alpha, \beta)}\right]\right\},
$$

where the Shannon-like integral functional of $\hat{P}_{n}^{(\alpha, \beta)}(x)$ is the following:

$$
\begin{aligned}
S\left[\hat{P}_{n}^{(\alpha, \beta)}\right] & =-\int_{-1}^{+1}\left[\hat{P}_{n}^{(\alpha, \beta)}(x)\right]^{2} h_{\alpha, \beta}^{J}(x) \ln \left\{\left[\hat{P}_{n}^{(\alpha, \beta)}(x)\right]^{2} h_{\alpha, \beta}^{J}(x)\right\} d x \\
& =E\left[\hat{P}_{n}^{(\alpha, \beta)}\right]+I\left[\hat{P}_{n}^{(\alpha, \beta)}\right],
\end{aligned}
$$


with the integral functional as follows [57,79]:

$$
\begin{aligned}
I\left[\hat{P}_{n}^{(\alpha, \beta)}\right] & =-\int_{-1}^{+1}\left[\hat{P}_{n}^{(\alpha, \beta)}(x)\right]^{2} h_{\alpha, \beta}^{J}(x) \ln h_{\alpha, \beta}^{J}(x) \\
& =-\alpha \psi(n+\alpha+1)-\beta \psi(n+\beta+1)+(\alpha+\beta) \\
& \times\left[-\ln 2+\frac{1}{2 n+\alpha+\beta+1}+2 \psi(2 n+\alpha+\beta+1)-\psi(n+\alpha+\beta+1)\right],
\end{aligned}
$$

and the Shannon entropy of $\hat{P}_{n}^{(\alpha, \beta)}(x)$ is given by the following:

$$
E\left[\hat{P}_{n}^{(\alpha, \beta)}\right]=-\int_{-1}^{+1}\left[\hat{P}_{n}^{(\alpha, \beta)}(x)\right]^{2} h_{\alpha, \beta}^{J}(x) \ln \left[\hat{P}_{n}^{(\alpha, \beta)}(x)\right]^{2} d x
$$

The explicit determination of this entropic functional for the Jacobi polynomials was not known up until now, except in the asymptotic case (whose value is given in the next section) and when the parameters $(\alpha, \beta)$ are equal to $(-1 / 2,-1 / 2)$ and $(1 / 2,1 / 2)$, which correspond to the families of Chebyshev polynomials of the first and second kind, denoted by $T_{n}(x)$ and $U_{n}(x)$, respectively. The following values were found $[10,80]$ :

$$
E\left[T_{n}\right]=-\frac{1}{\pi} \int_{-1}^{+1} T_{n}^{2}(x) \ln T_{n}^{2}(x) \frac{d x}{\sqrt{1-x^{2}}}=\ln 2-1 ; \quad \text { for } \quad n \geq 1
$$

for the Shannon entropy of the orthogonal Chebyshev polynomials of the first kind, and the values [80]

$$
E\left[U_{n}\right]=-\frac{2}{\pi} \int_{-1}^{+1} U_{n}^{2}(x) \ln U_{n}^{2}(x) \sqrt{1-x^{2}} d x=\frac{1}{n+1}-1 ; \quad \text { for } \quad n \geq 0
$$

for the Shannon entropy of the orthogonal Chebyshev polynomials of the second kind. Moreover, when the parameters $(\alpha, \beta)$ are equal to $(\lambda-1 / 2, \lambda-1 / 2)$ the corresponding Jacobi polynomials conform to the family of Gegenbauer polynomials $C_{n}^{(\lambda)}(x)$. Then, it is known the value for the Shannon entropy of the modified Gegenbauer polynomials $G_{n}^{(\lambda=2)}(x)$, which are the following polynomials:

$$
G_{k}^{(\lambda)}(x)=\left(\frac{k !(k+\lambda) \Gamma(2 \lambda)}{\lambda \Gamma(k+2 \lambda)}\right)^{\frac{1}{2}} C_{k}^{(\lambda)}(x)=\gamma_{k}^{\lambda} x^{k}+\text { lower degree terms }
$$

orthogonal with respect to the positive unit weight on $[-1,+1]$,

$$
h_{\lambda}(x)=\frac{\Gamma(\lambda+1)}{\sqrt{\pi} \Gamma(\lambda+1 / 2)}\left(1-x^{2}\right)^{\lambda-\frac{1}{2}} .
$$

Buyarov et al. [81] (see also [11,82]) found the following:

$$
\begin{array}{r}
E\left[G_{k}^{(\lambda)}\right]=-\int_{-1}^{+1}\left[G_{k}^{(\lambda)}(x)\right]^{2} \ln \left[G_{k}^{(\lambda)}(x)\right]^{2} h_{\lambda}(x) d x \\
=-\ln \left(\frac{3(n+1)}{n+3}\right)-\frac{n^{3}-5 n^{2}-29 n-27}{(n+1)(n+2)(n+3)}-\frac{1}{n+2}\left(\frac{n+3}{n+1}\right)^{n+2}, \quad \text { for } \lambda=2 .
\end{array}
$$

This expression was extended for Gegenbauer polynomials with integer order $\lambda$ (see $[81,83]$ and the references therein).

In the general case, the Shannon entropy of the orthonormal polynomials $\hat{p}_{n}(x)$ on the real line can be expressed in terms of the logarithmic potential of its Rakhmanov density $\rho_{n}(x)=\hat{p}_{n}^{2}(x) h(x)$ and, equivalently, by means of the mutual logarithmic energy 
between the normalized zero counting distribution $\lambda_{n}(x)=\frac{1}{n} \sum_{j=1}^{n} \delta\left(x-x_{j, n}\right)$ and the Rakhmanov density $\rho_{n}(x)$. Indeed, let $x_{j, n}(j=1, \ldots, n)$ be the zeros of $\hat{p}_{n}(x)$ and $\gamma_{n}$ the leading coefficient, so that the following holds:

$$
\left.\hat{p}_{n}(x)\right)=\gamma_{n} \prod_{j=1}^{n}\left(x-x_{j, n}\right), \quad \gamma_{n}>0 .
$$

Then, it can be proved that the Shannon entropy of the orthonormal polynomials $\hat{p}_{n}(x)$ can be expressed [80] as follows:

$$
E\left[\hat{p}_{n}\right]:=-2 \ln \gamma_{n}+2 n \int \mathcal{V}\left(x ; \lambda_{n}\right) \hat{p}_{n}^{2}(x) h(x) d x
$$

where

$$
\mathcal{V}(z ; \mu)=\int \ln \frac{1}{|z-x|} d \mu(x)
$$

is known as the logarithmic potential of the probability measure $\mu$ [84]. Moreover, taking into account the mutual logarithmic energy of the two measures $\mu$ and $\nu$, given [84] by

$$
I[v, \mu]=\int \mathcal{V}(z ; v) d \mu(x)=-\iint \ln |z-t| d v(t) d \mu(t),
$$

one has that the Shannon entropy of the orthonormal polynomials can be written [80] as follows:

$$
E\left[\hat{p}_{n}\right]=-2 \ln \gamma_{n}+2 n I\left[\lambda_{n}, \rho_{n}\right]
$$

When the support interval of $\mu(x)$ is compact, the potential $\mathcal{V}(z ; \mu)$ oscillates around its Robin (or extremal) constant. The zeros $x_{j, n}$ turn out to be [80] points of local minima for the potential $\mathcal{V}\left(x ; \rho_{n}\right)$; this implies that to calculate $E\left[\hat{p}_{n}\right]$, we have to sum up the values of the logarithmic potential $\mathcal{V}\left(x ; \rho_{n}\right)$ at its local minima. Then, by applying this expression to the Jacobi polynomials and taking into account Fubini's theorem, one finally has the Shannon entropy of the orthonormal Jacobi polynomials $\hat{P}_{n}^{(\alpha, \beta)}(x)$ :

$$
E\left[\hat{P}_{n}^{(\alpha, \beta)}\right]:=-2 \ln \gamma_{n}-2 \sum_{j=1}^{n} \mathcal{V}\left(x_{j, n} ; \rho_{n, \alpha, \beta}\right),
$$

in terms of the following logarithmic potential evaluated at the zeros of the polynomial:

$$
\mathcal{V}\left(z ; \rho_{n, \alpha, \beta}\right)=\int \hat{P}_{n}^{(\alpha, \beta)}(x)^{2} h_{\alpha, \beta}^{J}(x) \ln \frac{1}{|z-x|} d x
$$

Similarly, one can obtain the Shannon entropy of the remaining HOPs in terms of the values of their logarithmic potentials evaluated at their zeros, such as the Hermite, Laguerre and Gegenbauer polynomials, as explained in detail in [11,36,80,85]. Additionally, the Shannon entropy of the HOPs was calculated [58] in terms of the eigenvalues of their associated Jacobi matrix and the ordinary moments (15) of their corresponding Rakhmanov density (1). In practice, however, we have a nontrivial task in both cases, especially when the degree of the polynomials is not sufficiently low. Then, it appears that the determination of the asymptotical $(n \rightarrow \infty)$ values of the Shannon entropy of the HOPs is necessary, which is shown in the next section.

Since the expressions obtained for the Shannon entropy $E\left[p_{n}\right]$ and, consequently, for the Shannon spreading lengths $\mathcal{L}_{1}^{S}\left[p_{n}\right]$ of the HOPs are not easily manipulated analytically, it is also interesting to obtain simple, compact and accurate upper bounds to these quantities in terms of the degree and the parameters of their weight function. Some upper bounds were found for Hermite [22], Laguerre [24] and Jacobi [23] polynomials by means of the ordinary moments of their associated Rakhmanov densities (1). In the Hermite and 
Laguerre cases, we used an optimized information-theoretical technique to obtain the upper bounds based on the non-negativity of the Kullback-Leibler functional of the Rakhmanov density of the polynomial and a probability density of exponentially decreasing type of the form $\left.\exp \left(-x^{k}\right)\right)$. It is found that the following holds:

$$
\mathcal{L}_{1}^{S}\left[\hat{H}_{n}\right] \leq \frac{2(e k)^{\frac{1}{k}}}{k} \Gamma\left(\frac{1}{k}\right)\left(\left\langle x^{k}\right\rangle_{n}\right)^{1 / k} ; \quad k=2,4, \ldots
$$

and

$$
\mathcal{L}_{1}^{S}\left[\hat{L}_{n}^{(\alpha)}\right] \leq \frac{(k e)^{\frac{1}{k}}}{k} \Gamma\left(\frac{1}{k}\right)\left(\left\langle x^{k}\right\rangle_{n, \alpha}\right)^{1 / k} ; \quad k>0,
$$

for the Shannon spreading length of Hermite and Laguerre polynomials, respectively, where the ordinary moments of such polynomials are given by (17) and (19). In the Jacobi case, beyond the general upper bound $\mathcal{L}_{1}^{S}\left[p_{n}\right] \leq 2$ valid for any probability density with the support interval $[-1,+1]$, some variational bounds were found by means of the following ordinary and logarithmic moments: $\langle x\rangle,\left\langle x^{2}\right\rangle,\left\langle\ln x^{2}\right\rangle,\langle\ln (1 \pm x)\rangle,\left\langle\ln \left(1-x^{2}\right)\right\rangle$. In particular, it is known that

$$
\mathcal{L}_{1}^{S}\left[\hat{P}_{n}^{(\alpha, \beta)}\right] \leq \frac{2 \sinh \left(\lambda_{2}\right)}{\lambda_{2}} \exp \left(-1-\lambda_{2} \operatorname{coth}\left(\lambda_{2}\right)\right),
$$

where $\lambda_{2}$ is given in terms of $\langle x\rangle_{n, \alpha, \beta}$ by means of the following implicit equation:

$$
\frac{1}{\lambda_{2}}-\operatorname{coth}\left(\lambda_{2}\right)=\langle x\rangle_{n, \alpha, \beta}
$$

where, according to (21), the first order moment $\langle x\rangle_{n, \alpha, \beta}$ of the Rakhmanov density $\rho_{n, \alpha, \beta}(x)$ of the Jacobi polynomials is given explicitly by the following:

$$
\langle x\rangle_{n, \alpha, \beta}=\frac{\beta^{2}-\alpha^{2}}{(2 n+\alpha+\beta)(2 n+\alpha+\beta+2)} .
$$

These variational bounds to $\mathcal{L}_{1}^{\mathcal{S}}\left[\hat{P}_{n}^{(\alpha, \beta)}\right]$ have been analytically and numerically discussed in detail [23].

Finally, let us also remark that Beckermann et al. [86] found some asymptotic upper bounds to the Shannon entropy $E\left[\hat{p}_{n}\right]$ for polynomials $\hat{p}_{n}(x)$ orthonormal with respect to a weight function $h(x)$ on the support $[-1,+1]$, which belongs to the Szegö class, although the determination of the entropy itself remains an open problem. See [43,87-89] for recent extensions of these results.

\section{Shannon Entropy $E\left[p_{n}\right]$ and $\mathfrak{L}_{q}$-Norms $\mathcal{N}_{q}\left[p_{n}\right]$ of HOPs Degree Asymptotics}

In this section, we tackle and solve the asymptotics $(n \rightarrow \infty)$ for the Shannon entropy of the three canonical families of the HOPs and the Freud polynomials (which is a generalization of the Hermite polynomials). This study was initiated in 1994 [33-35] and reviewed in 2001 [11] and 2010 [36].

The following approach was used. Taking into account the limiting relation (3), we can convert the calculation of this Shannon entropy of the HOPs to the evaluation of the (unweighted) $\mathfrak{L}_{q}$-norms $\mathcal{N}_{q}\left[\hat{p}_{n}\right]$ since the following holds:

$$
E\left[\hat{p}_{n}\right]=-\lim _{q \rightarrow 1} \frac{1}{q-1} \ln \int\left|\hat{p}_{n}(x)\right|^{2 q} h(x) d x=-\lim _{q \rightarrow 1} \frac{\partial}{\partial q} \mathcal{N}_{2 q}\left[\hat{p}_{n}\right],
$$

where

$$
\mathcal{N}_{q}\left[\hat{p}_{n}\right]:=\int\left|\hat{p}_{n}(x)\right|^{q} h(x) d x
$$


denotes the (unweighted) $\mathfrak{L}_{q}(h)$ norms of $\hat{p}_{n}(x)$. Then, the asymptotical value for the Shannon entropy $E\left[\hat{p}_{n}\right]$ is as follows $[34,36]$ :

$$
E_{\infty}=\lim _{n \rightarrow \infty} E\left[\hat{p}_{n}\right]=-\left.\lim _{n \rightarrow \infty} \frac{\partial \mathcal{N}_{2 q}\left[\hat{p}_{n}\right]}{\partial q}\right|_{q=1}=-\left.\mathcal{N}_{2 q}^{\prime}\right|_{q=1}
$$

This approach based on the (unweighted) $\mathfrak{L}_{q}$-norms can be used for the HOPs with both bounded and unbounded supports. However, for an absolutely continuous weight function $h(x)$ on the real line, it is more convenient to calculate the Shannon entropy $E\left[\hat{p}_{n}\right]$ from the weighted $\mathfrak{L}_{q}$-norms $W_{q}\left[\hat{p}_{n}\right]$ given by (30) so that, according to (91) and (89), one has the following [34]:

$$
E\left[\hat{p}_{n}\right]=S\left[\hat{p}_{n}\right]-I\left[\hat{p}_{n}\right]=-\left.\frac{\partial W_{q}\left[\hat{p}_{n}\right]}{\partial q}\right|_{q=1}-I\left[\hat{p}_{n}\right] .
$$

Thus, the asymptotical value $E_{\infty}$ for the Shannon entropy is basically controlled by the asymptotics of $W_{q}\left[\hat{p}_{n}\right]$. Let us now apply this second approach to the Freud polynomials $F_{n}^{(\kappa)}(x)$, which are orthogonal with respect to the weight function $h_{\kappa}(x)=e^{-|x|^{\kappa}}, \kappa>1$, $\infty<x<+\infty$. In this case we have that the Shannon entropy is the following:

$$
E\left[F_{n}^{(\kappa)}\right]=-\left.\frac{\partial W_{q}\left[F_{n}^{(\kappa)}\right]}{\partial q}\right|_{q=1}-I\left[F_{n}^{(\kappa)}\right]=-\left.\frac{\partial W_{q}\left[F_{n}^{(\kappa)}\right]}{\partial q}\right|_{q=1}-\frac{2 n+1}{\kappa}
$$

Now, the asymptotical behavior $(n \rightarrow \infty)$ of the weighted $\mathfrak{L}_{q}$-norms $W_{q}\left[F_{n}\right]$ is found to be the following:

$$
W_{q}\left[\hat{F}_{n}^{(\kappa)}\right]=\left(\frac{2}{\pi}\right)^{q} \frac{\Gamma(q+1 / 2)}{\Gamma(q+1)} \frac{\Gamma(1-q / 2)}{\Gamma(3 / 2-q / 2)} \alpha_{n}^{1-q}(1+o(1))
$$

with

$$
\alpha_{n}=\left(\frac{2 n+1}{2 \beta}\right)^{1 / \kappa} \text { and } \beta=\frac{\Gamma(\kappa / 2+1 / 2)}{\Gamma(1 / 2) \Gamma(\kappa / 2)} .
$$

Then, from the last three expressions, we have that the $(n \rightarrow \infty)$-asymptotics of the Shannon entropy of the Freud polynomials [35] is given by the following:

$$
E\left[\hat{F}_{n}^{(\kappa)}\right]=-\frac{2 n+1}{\kappa}+\frac{1}{\kappa} \ln (2 n)-\frac{1}{\kappa} \ln \left(\frac{\sqrt{\pi} \Gamma(\kappa / 2)}{2 \Gamma(\kappa / 2+1 / 2)}\right)+\ln \pi-1+o(1)
$$

Consequently, for $\kappa=2$ we have that the asymptotics $(n \rightarrow \infty)$ for the Shannon entropy of the Hermite polynomials $H_{n}(x)$ is given by the following:

$$
E\left[\hat{H}_{n}\right]=-n+\ln \sqrt{2 n}-\frac{3}{2}+\ln \pi+o(1)
$$

The same technique can be used to find the asymptotic behaviour $(n \rightarrow \infty)$ for the Shannon entropy of the orthonormal Laguerre polynomials $\hat{L}_{n}^{(\alpha)}(x)$, obtaining [90] for fixed real $\alpha>-1$ such that the following holds:

$$
E\left[\hat{L}_{n}^{(\alpha)}\right]=-2 n+(\alpha+1) \ln n-\alpha-2+\ln \pi+o(1)
$$

Similar operations with the orthonormal Jacobi polynomials $\hat{P}_{n}^{(\alpha, \beta)}(x)$ by using the (unweighted) $\mathfrak{L}_{q}$-norms $\mathcal{N}_{q}\left[\hat{p}_{n}\right]$ given by (109) allowed us, according to (108), to find the following expression:

$$
E\left[\hat{P}_{n}^{(\alpha, \beta)}\right]=-2 n+(\alpha+1) \ln n-\alpha-2+\ln \pi+o(1)
$$


for the asymptotic behavior of the Shannon entropy of the orthonormal Jacobi polynomials. A particularly relevant family of the Jacobi polynomials are the Gegenbauer polynomials $C_{n}^{(\lambda)}(x)$, which are orthogonal with respect to the weight function $h_{\lambda}(x)=\left(1-x^{2}\right)^{\lambda-\frac{1}{2}}$ on the interval $[-1,+1]$; then, the orthonormal Gegenbauer polynomial $\hat{C}_{n}^{(\lambda)}(x)$ are as follows:

$$
\widetilde{C}_{n}^{(\lambda)}(x)=\frac{C_{n}^{(\lambda)}}{\kappa_{n}} ; \quad \text { with } \quad \kappa_{n}^{2}=\frac{2^{1-2 \lambda} \pi \Gamma(n+2 \lambda)}{[\Gamma(\lambda)]^{2}(n+\lambda) n !},
$$

so that, according to (109), the Shannon entropy of $\hat{C}_{n}^{(\lambda)}(x)$ has the following asymptotic expression [34]:

$$
E\left(\hat{C}_{m}^{\alpha}\right) \equiv-\int_{-1}^{+1} h_{\alpha}(x)\left[\hat{C}_{m}^{\alpha}(x)\right]^{2} \ln \left[\hat{C}_{m}^{\alpha}(x)\right]^{2} d x=\ln \pi+(1-2 \alpha) \ln 2-1+o(1),
$$

for fixed $\lambda$ and large degree $n$. This asymptotic expression was improved by obtaining further terms as shown in $[81,83]$. Further details about the proof of these results and their extensions to more general orthonormal polynomials belonging to the Bernstein and Szegö classes can be seen in $[34,36,86-89]$.

Moreover, from the previous expressions (115)-(117) for the asymptotics of the Shannon entropy $E\left[\hat{p}_{n}\right]$ of HOPS and the exact expressions (94), (97) and (100) for the integrals $I\left[\hat{p}_{n}\right]$ given by (89), we have the following asymptotics $(n \rightarrow \infty)$ for the Shannon-like integral functionals $S\left[\hat{p}_{n}\right]$ of the HOPS [34]:

$S\left[\hat{H}_{n}\right]=\ln \sqrt{2 n}+\ln \pi-1+o(1), \quad S\left[\hat{L}_{n}^{(\alpha)}\right]=(\alpha+1) \ln n-\alpha \psi(\alpha+n+1)-1+\ln (2 \pi)+o(1)$,

and

$$
S\left[\hat{P}_{n}^{(\alpha, \beta)}\right]=\ln \pi-1+o(1)
$$

for the Hermite, Laguerre and Jacobi polynomials, respectively. To obtain the last expression, we have also taken into account that $\lim _{n \rightarrow \infty} I\left[\widetilde{P}_{n}^{(\alpha, \beta)}\right]=(\alpha+\beta) \ln 2$. Then, the Shannon spreading length $\mathcal{L}_{1}^{S}\left[\hat{p}_{n}\right]=\mathcal{L}_{1}^{S}\left[\rho_{n}\right]$ of the three canonical families of the HOPs $\left\{\hat{p}_{n}(x)\right\}$ have the following asymptotical values:

$$
\mathcal{L}_{1}^{S}\left[\hat{H}_{n}\right] \simeq \frac{\pi}{e} \sqrt{2 n}, \quad \mathcal{L}_{1}^{S}\left[\hat{L}_{n}^{(\alpha)}\right] \simeq \frac{\pi}{e} 2 n, \quad \text { and } \quad \mathcal{L}_{1}^{S}\left[\hat{P}_{n}^{(\alpha, \beta)}\right] \simeq \frac{\pi}{e}
$$

for Hermite, Laguerre and Jacobi polynomials, respectively. By comparing these expressions with the asymptotical values of the standard deviation $\Delta x$ given by Equations (18), (20) and (22) for Hermite, Laguerre and Jacobi polynomials, respectively, one is led to the following linear relation:

$$
\mathcal{L}_{1}^{S}\left[\hat{p}_{n}\right] \simeq \frac{\pi \sqrt{2}}{e} \Delta x\left[\hat{p}_{n}\right] \simeq 1.6389 \Delta x\left[\hat{p}_{n}\right], \quad n \rightarrow \infty
$$

which holds for the three canonical families of HOPs [91] (see also [22-24]); note that it fulfills the general Cramér-Rao relation (9). In fact, the validity of this relation for HOPs extends to the whole class of Bernstein-Szegö polynomials, although it fails for arbitrary orthogonal polynomials. For example, it is violated for Freud polynomials for which the Shannon length and the standard deviation have a quadratic relation [91].

\section{Conclusions}

Hypergeometric orthogonal polynomials (HOPs) are the simplest non-elementary functions in the theory of special functions of applied mathematics and mathematical physics. The three canonical families of HOPs (Hermite, Laguerre and Jacobi) and their generalizations are not only interesting per se, but also because of their physico-mathematical applications in science and technology. In particular, they have been shown to control the physical solutions (wave functions) of the Schrödinger equation of numerous classical and quantum systems, which characterize, for example, the bound stationary states of systems 
subject to spherically-symmetric quantum-mechanical potentials (harmonic systems of oscillator type, Coulomb systems of hydrogenic type, etc.).

Keeping this in mind, we have examined and reviewed in this work the present knowledge of the spreading of the HOPs along the orthogonality support of their weight function by means of the entropy-like measures of Fisher, Shannon and Rényi types (and their associated $\mathfrak{L}_{q}$-norms) - far beyond the ordinary moments and the standard deviation of their associated Rakhmanov probability density. We have shown the analytical expressions that allow one to compute such spreading measures of the HOPs in a numerical and symbolic manner by means of the degree and the weight function's parameters of the polynomials. In addition, we have shown the asymptotic behavior $(n \rightarrow \infty)$ for the $\mathfrak{L}_{q}$-norms of the HOPs $\left\{p_{n}(x)\right\}$ and some generalizations, which provides simple and compact expressions for the first dominant terms of these quantities in terms of $q$ and the weight parameters. The latter controls the physical entropies of the highly excited states of atomic systems and the corresponding Rydberg logical gates of quantum computation.

As always, some related open issues deserve to be mentioned, including the following: the calculation of the upper bounds to the Rényi spreading lengths $\mathcal{L}_{q}^{R}\left[p_{n}\right]$ for all $q$ and $n$, extending the corresponding quantities obtained for the Shannon spreading length as mentioned above; the algebraic determination of the Shannon entropies for the HOPs at all $n$ by means of a technique that does not require the evaluation of the logarithmic potential evaluated at the zeros of the polynomials, perhaps at the cost of evaluating some generalized hypergeometric functions at unity (see e.g., [92]); and the asymptotics of the (unweighted) $\mathfrak{L}_{q}$-norms of HOPs when $n \rightarrow \infty$ and when the parameters of the weight function become large or very large. In addition, the asymptotics of the Shannon entropy of orthogonal polynomials in the whole Szegö class remains open, although some recent relevant efforts relative to asymptotic upper bounds and universality limits were carried out (see $[43,86,89])$.

Funding: This research received no external funding.

Institutional Review Board Statement: Not applicable.

Informed Consent Statement: Not applicable.

Data Availability Statement: Not applicable.

Acknowledgments: This work was partially supported by the Agencia Estatal de Investigación (Spain) and the European Regional Development Fund (FEDER) under the grant PID2020-113390GB-I00.

Conflicts of Interest: The author declares no conflict of interest.

\section{References}

1. Szegö, G. Orthogonal Polynomials; American Mathematical Society: Providence, RI, USA, 1975.

2. Chihara, T.S. An Introduction to Orthogonal Polynomials; Gordon and Breach: New York, NY, USA, 1978

3. Nikiforov, A.F.; Uvarov, V.B. Special Functions of Mathematical Physics; Birkhüser: Basel, Switzerland, 1988.

4. Temme, N.M. Special Functions: An Introduction to the Classical Functions of Mathematical Physics; Wiley-Intersciente: New York, NY, USA, 1996.

5. Ismail, M.E.H. Classical and Quantum Orthogonal Polynomials in One Variable; Encyclopedia for Mathematics and Its Applications; Cambridge University Press: Cambridge, UK, 2005.

6. Olver, F.W.J.; Lozier, D.W.; Boisvert, R.F.; Clark, C.W. NIST Handbook of Mathematical Functions; Cambridge University Press: Cambridge, UK, 2010.

7. Koekoek, R.; Lesky, P.A.; Swarttouw, R.F. Hypergeometric Orthogonal Polynomials and Their q-Analogues; Springer: Berlin, Germany, 2010.

8. Andrews, G.E.; Askey, R.; Roy, R. Special Functions; Encyclopedia for Mathematics and Its Applications; Cambridge University Press: Cambridge, UK, 1999.

9. Rakhmanov, E.A. On the asymptotics of the ratio of orthogonal polynomials. Math. USSR-Sb. 1977, 32, 199-213. [CrossRef]

10. Yáñez, R.J.; Van Assche, W.; Dehesa, J.S. Position and momentum information entropies of the D-dimensional harmonic oscillator and hydrogen atom. Phys. Rev. A 1994, 50, 3065. [CrossRef]

11. Dehesa, J.S.; Martínez-Finkelshtein, A.; Sánchez-Ruiz, J. Quantum information entropies and orthogonal polynomials. J. Comput. Appl. Math. 2001, 133, 23-46. [CrossRef] 
12. Bagrov, V.G.; Gitman, D.M. Exact Solutions of Relativistic Wavefunctions; Kluwer Academic Publishers: Dordrecht, The Netherlands, 1990.

13. Cooper, F.; Khare, A.; Sukhature, U. Supersymmetry in Quantum Mechanics; World Scientific Publishing: London, UK, 2001.

14. Cover, T.M.; Thomas, J.A. Elements of Information Theory; Wiley: New York, NY, USA, 1991.

15. Nielsen, M.A.; Chuang, I.L. Quantum Computation and Quantum Information, 2nd ed.; Cambridge University Press: Cambridge, UK, 2000.

16. Bruss, D.; Leuchs, G. Quantum Information: From Foundations to Quantum Technology; Wiley-VCH: Weinheim, Germany, 2019.

17. Fisher, R.A. Theory of statistical estimation. Proc. Camb. Philos. Soc. 1925, 22, 700. [CrossRef]

18. Frieden, B.R. Science from Fisher Information; Cambridge University Press: Cambridge, UK, 2004.

19. Shannon, C. A mathematical theory of communication. Bell Syst. Tech. J. 1948, 27, 379. [CrossRef]

20. Rényi A. On measures of information and entropy. In Proceedings of the 4th Berkeley Symposium on Mathematical Statistics and Probability; University of California Press: Berkeley, CA, USA, 1961; Volume 1, pp. 547-561.

21. Rényi, A. Probability Theory; North-Holland Publishing Company: Amsterdam, The Netherlands, 1970.

22. Sánchez-Moreno, P.; Dehesa, J.S.; Manzano, S.; Yáñez, R.J. Spreading lengths of Hermite polynomials. J. Comput. Appl. Math. 2010, 233, 2136. [CrossRef]

23. Guerrero, A.; Sánchez-Moreno, P.; Dehesa, J.S. Information-theoretic lengths of Jacobi polynomials. J. Phys. A Math. Theor. 2010, 43, 305203. [CrossRef]

24. Sánchez-Moreno, P.; Manzano, D.; Dehesa, J.S. Direct spreading measures of Laguerre polynomials. J. Comput. Appl. Math. 2011, 235, 1129. [CrossRef]

25. Dehesa, J.S.; Guerrero, A.; Sánchez-Moreno, P. Information-theoretic-based spreading measures of orthogonal polynomials. Complex Anal. Oper. Theory 2012, 6, 585-601. [CrossRef]

26. Hall, M.J.W. Universal geometric approach to uncertainty, entropy and information. Phys. Rev. A 1999, 59, 2602-2615. [CrossRef]

27. Onicescu, O. Theorie de l'information. Energie informationelle. C. R. Acad. Sci. Paris A 1966, 263, A841.

28. Aczel, J.; Daroczy, Z. On Measures of Information and Their Characterizations; Academic Press: New York, NY, USA, 1975.

29. Leonenko N.;, Pronzato L.; Savani V. A class of Rényi estimators for multidimensional densities. Ann. Stat. 2008, 40, 2153. [CrossRef]

30. Dembo, A.; Cover, T.M.; Thomas, J.A. Information-theoretic inequalities. IEEE Trans. Inf. Theory 1991, 37, 1501. [CrossRef]

31. Zyczkowski, K. Rényi extrapolation of Shannon entropy. Open Syst. Inf. Dyn. 2003, 10, 297. [CrossRef]

32. Gyftopoulos, E.P.; Cubukcu, E. Thermodynamic definition and quantum expression. Phys. Rev. E 2008, 55, 3851. [CrossRef]

33. Aptekarev, A.I.; Dehesa, J.S.; Yáñez, R.J. Spatial entropy of central potentials and strong asymptotics of orthogonal polynomials. J. Math. Phys. 1994, 35, 4423-4428. [CrossRef]

34. Aptekarev, A.I.; Buyarov, V.; Dehesa, J.S. Asymptotic behavior of the $L_{p}$-norms and the entropy for general orthogonal polynomials. Russ. Acad. Sci. Sbornik Math. 1994, 85, 3-30; English translation Russ. Acad. Sci. Sb. Math. 1995, 82, $373-395$.

35. Van Assche, W.; Yáñez, R.J.; Dehesa, J.S. Entropy of orthogonal polynomials with Freud weights and information entropies of the harmonic oscillator potential. J. Math. Phys. 1995, 36, 4106-4118. [CrossRef]

36. Aptekarev, A.I.; Dehesa, J.S.; Martinez-Finkelshtein, A. Asymptotics of orthogonal polynomial's entropy. J. Comput. Appl. Math. 2010, 233, 1355-1365. [CrossRef]

37. Dehesa, J.S.; Toranzo, I.V. Dispersion and entropy-like measures of multidimensional harmonic systems: Application to Rydberg states and high-dimensional oscillators. Eur. Phys. J. Plus 2020, 135, 721. [CrossRef]

38. Dehesa, J.S.; Toranzo, I.V.; Puertas-Centeno, D. Entropic measures of Rydberg-like harmonic states. Int. J. Quantum Chem. 2017, 117, 48-56. [CrossRef]

39. Dehesa, J.S.; López-Rosa, S.; Martínez-Finkelshtein, Y.R.J. Information Theory of D-Dimensional Hydrogenic Systems: Application to Circular and Rydberg States. Int. J. Qunatum Chem. 2010, 110, 1529-1548. [CrossRef]

40. Toranzo, I.V.; Puertas-Centeno, D.; Dehesa, J.S. Entropic properties of D-dimensional Rydberg systems. Physica A 2016, 462, 1197-1206. [CrossRef]

41. Toranzo, I.V.; Dehesa, J.S.; Rényi, S. Tsallis entropies of Rydberg hydrogenic systems. EPL (Europhys. Lett.) 2016, $113,48003$. [CrossRef]

42. Levin, E.; Lubinsky, D.S. Universality limits in the bulk for varying measures. Adv. Math. 2008, 219, 743-779. [CrossRef]

43. Levin, E.; Lubinsky, D.S. Universality Limits and Entropy Integrals. In Bounds and Asymptotics for Orthogonal Polynomials for Varying Weights; Springer Briefs in Mathematics; Springer: Cham, Switzerland, 2018.

44. Aptekarev, A.I.; Dehesa, J.S.; Martínez-Finkelshtein, A.; Yáñez, R.J. Discrete entropies of orthogonal polynomials. Const. Approx. 2009, 30, 93-119. [CrossRef]

45. Martínez-Finkelshtein, A.; Nevai, P.; Peña, A. Discrete entropy of generalized Jacobi polynomials. J. Math. Anal. Appl. 2015, 431, 99-110. [CrossRef]

46. Sfetcu, R.C. Tsallis and Rényi divergences of generalized Jacobi polynomials. Physica A 2016, 460, 131-138. [CrossRef]

47. Kendall, M.G.; Stuart, A. The Advanced Theory of Statistics; Charles Griffin Co.: London, UK, 1969; Volume 1.

48. Sichel, H.S. Fitting growth and frequency curves by the method of frequency moments. J. R. Stat. Soc. A 1947, 110, 337-347. [CrossRef]

49. Yule, G.U. On some properties of the normal distribution, univariate and bivariate, based on the sum of squares of frequencies. Biometrika 1938, 30, 1-10. [CrossRef] 
50. Shohat, J.A.; Tamarkin, J.D. The Problem of Moments; Mathematical Surveys Volume 1; American Mathematical Society: New York, NY, USA, 1943.

51. Shenton, L.R. Efficiency of the method of moments and the Gram-Charlier type A distribution. Biometrika 1951, 38, 58. [CrossRef]

52. Hall, M.J.W. Quantum properties of classical Fisher information. Phys. Rev. A 2000, 62, 012107. [CrossRef]

53. Hall, M.J.W. Exact uncertainty relations. Phys. Rev. A 2001, 64, 052103. [CrossRef]

54. Kennard, E.H. Zur Quantenmechanik einfacher Bewegungstypen. Z. Phys. 1927, 44, 326. [CrossRef]

55. Bialynicki-Birula, I.; Mycielski, J. Uncertainty relations for information entropy in wave mechanics. Commun. Math. Phys. 1975, 44, 129. [CrossRef]

56. Zozor, S.; Portesi, M.; Vignat, C. Some extensions of the uncertainty principle. Physica A 2008, 387, 4800-4808. [CrossRef]

57. Dehesa, J.S.; Sánchez-Moreno, P.; Yáñez, R.J. Cramér-Rao information plane of orthogonal hypergeometric polynomials. J. Comput. Appl. Math. 2006, 186, 523-541. [CrossRef]

58. He, M.X.; Ricci, P.E. Information entropy of orthogonal polynomials. Appl. Math. Comput. 2002, 128, 261-274. [CrossRef]

59. Dehesa, J.S.; Moreno-Balcázar, J.J.; Toranzo, I.V. Linearization and Krein-like functionals of hypergeometric orthogonal polynomials. J. Math. Phys. 2018, 59, 123504. [CrossRef]

60. Sánchez-Ruiz, J.; Dehesa, J.S. Fisher information of orthogonal hypergeometric polynomials. J. Comput. Appl. Math. 2005, 182, 150-164. [CrossRef]

61. Yáñez, R.J.; Sánchez-Moreno, P.; Zarzo, A.; Dehesa, J.S. Fisher information of special functions and second-order differential equations. J. Math. Phys. 2008, 49, 082104. [CrossRef]

62. Bernstein, S. Complete Works; The Academy of Sciences of the USSR: Moscow, Russia, 1954; Volume 2.

63. Suetin, P.K. V. A. Steklov's problem in the theory of orthogonal polynomials. J. Soviet. Math. 1979, 12, 631-682. [CrossRef]

64. Lubinsky, D.S.; Saff, E.B. Strong asymptotics for extremal polynomials associated with weights on $\mathbb{R}$; In Lecture Notes in Mathematics; Springer: Belin, Germany, 1988; Volume 1305.

65. Zygmund, A. Trigonometric Series; Cambridge University Press: Cambridge, UK, 2002.

66. Comtet, L. Advanced Combinatorics; D. Reidel Publishing Company: Dordrecht, The Netherlands, 1974.

67. Srivastava, H.M. A unified theory of polynomial expansions and their applications involving Clebsch-Gordan type linearization relations and Neumann series. Astrophys. Space Sci. 1988, 150, 251-266. [CrossRef]

68. Srivastava, H.M.; Niukkanen, A.W. Some Clebsch-Gordan type linearization relations and associated families of Dirichlet integrals. Math. Comput. Model. 2003, 37, 245-250. [CrossRef]

69. Srivastava, H.M.; Karlsson, P.W. Multiple Gaussian Hypergeometric Series; Wiley: New York, NY, USA, 1985.

70. Sánchez-Moreno, P.; Dehesa, J.S.; Zarzo, A.; Guerrero, A. Rényi entropies, $\mathfrak{L}_{q}$ norms and linearization of powers of hypergeometric orthogonal polynomials by means of multivariate special functions. Appl. Math. Comput. 2013, 223, 25-33.

71. Puertas-Centeno, D.; Toranzo, I.V.; Dehesa, J.S. Exact Rényi entropies of D-dimensional harmonic systems. Eur. Phys. J. Spec. Top. 2018, 227, 345-352. [CrossRef]

72. Puertas-Centeno, D.; Toranzo, I.V.; Dehesa, J.S. Rényi entropies for multidimensional hydrogenic systems in position and momentum spaces. J. Stat. Mech. 2018, 2018, 073203. [CrossRef]

73. Deift, P.; Kriecherbauer, T.; McLaughlin, K.T.R.; Venakides S.; Zhou, X. Strong asymptotics of orthogonal polynomials with respect to exponential weight. Comm. Pure Appl. Math. 1999, 52, 1491-1552. [CrossRef]

74. Deift, P. Orthogonal Polynomials and Random Matrices: A Riemann-Hilbert Approach, Courant Lecture Notes in Mathematics; American Mathematical Society: Providence, RI, USA, 1999; Volume 3.

75. Tulyakov, D.N. Plancherel-Rotach type asymptotics for solutions of linear recurrence relations with rational coefficients. Russian Acad. Sci. Sb. Math. 2010, 201, 1355-1402. [CrossRef]

76. Aptekarev, A.I.; Dehesa, J.S.; Sánchez-Moreno, P.; Tulyakov, D.N. Asymptotics of $L_{p}$-norms of Hermite polynomials and Rényi entropy of Rydberg oscillator states. Contemp. Math. 2012, 578, 19-29.

77. Aptekarev, A.I.; Tulyakov, D.N.; Toranzo, I.V.; Dehesa, J.S. Rényi entropies of the highly-excited states of multidimensional harmonic oscillators by use of strong Laguerre asymptotics. Eur. Phys. J. B 2016, 89, 85 [CrossRef]

78. Aptekarev, A.I.; Belega, E.D.; Dehesa, J.S. Rydberg multidimensional states: Rényi and Shannon entropies in momentum space. J. Phys. A Math. Theor. 2021, 54, 035305. [CrossRef]

79. Sánchez-Ruiz, J.; Dehesa, J.S. Entropic integrals of orthogonal hypergeometric polynomials with general supports. J. Comput. Appl. Math. 2000, 118, 311-322. [CrossRef]

80. Dehesa, J.S.; Van Assche, W.; Yáñez, R.J. Information entropy of classical orthogonal polynomials and their application to the harmonic oscillator and Coulomb potentials. Methods Appl. Anal. 1997, 4, 91-110. [CrossRef]

81. Buyarov, V.S.; López-Artés, P.; Martínez-Finkelshtein, A.; Van Assche W. Information entropy of Gegenbauer polynomials. J. Phys. A Math. Gen. 2000, 33, 6549-6560. [CrossRef]

82. Sánchez-Ruiz, J. Information entropy of Gegenbauer polynomials and Gaussian quadrature. J. Phys. A Math. Gen. 2003, 36, 4857-4865. [CrossRef]

83. De Vicente, J.I.; Gandy, S.; Sánchez-Ruiz, J. Information entropy of Gegenbauer polynomials of integer parameter. J. Phys. A Math. Theor. 2007, 40, 8345-8361. [CrossRef]

84. Nikishin, E.M.; Sorokin, V.N. Rational Approximations and Orthogonality; Translations of Mathematical Monographs 92; American Mathematical Society: Providence, RI, USA, 1991. 
85. Sánchez-Ruiz, J. Logarithmic potential of Hermite polynomials and information entropies of the harmonic oscillator eigenstates. J. Math. Phys. 1997, 38, 5031-5043. [CrossRef]

86. Beckermann, B.; Martínez-Finkelshtein, A.; Rakhmanov, E.A.; Wielonsky, F. Asymptotic upper bounds for the entropy of orthogonal polynomials in the Szegö class. J. Math. Phys. 2004, 45, 4239-4254. [CrossRef]

87. Denisov, S.; Kupin, S. On the growth of the polynomial entropy integrals for measures in the Szegö class. Adv. Math. 2013, 241, 18-32. [CrossRef]

88. Levin, E.; Lubinsky, D.S. Asymptotics for entropy integrals associated with exponential weights. J. Comp. Appl. Math. 2003, 156, 265-283. [CrossRef]

89. Alexis, M.; Aptekarev, A.I.; Denisov, S. Continuity of weighted operators, Muckenhoupt $A_{p}$ weights and Steklov problem for orthogonal polynomials. arXiv 2019, arXiv:1912.09377.

90. Dehesa, J.S.; Yáñez, R J.; Aptekarev, A.I.; Buyarov, V. Strong asymptotics of Laguerre polynomials and information entropies of two-dimensional harmonic oscillator and one-dimensional Coulomb potentials. J. Math. Phys. 1998, 39, 3050-3060. [CrossRef]

91. De Vicente, J.I.; Sánchez-Ruiz, J.; Dehesa, J.S. Information entropy and standard deviation of probability distributions involving orthogonal polynomials. In Proceedings of the Contribution to IWOP 2004, Madrid, Spain, 11 March 2004.

92. Toranzo, I.V.; Puertas-Centeno, D.; Sobrino, N.; Dehesa, J.S. Analytical Shannon information entropies for all discrete multidimensional hydrogenic states. Int. J. Quantum Chem. 2020, 120, e26077. [CrossRef] 